\title{
Analytic dependence of a periodic analog of a fundamental solution upon the periodicity parameters
}

\author{
M. Lanza de Cristoforis ${ }^{1}$ - P. Musolino $^{2}$
}

Received: 2 May 2017 / Accepted: 1 November 2017 / Published online: 24 November 2017

(C) Fondazione Annali di Matematica Pura ed Applicata and Springer-Verlag GmbH Germany, part of Springer Nature 2017

\begin{abstract}
We prove an analyticity result in Sobolev-Bessel potential spaces for the periodic analog of the fundamental solution of a general elliptic partial differential operator upon the parameters which determine the periodicity cell. Then we show concrete applications to the Helmholtz and the Laplace operators. In particular, we show that the periodic analogs of the fundamental solution of the Helmholtz and of the Laplace operator are jointly analytic in the spatial variable and in the parameters which determine the size of the periodicity cell. The analysis of the present paper is motivated by the application of the potential theoretic method to periodic anisotropic boundary value problems in which the "degree of anisotropy" is a parameter of the problem.
\end{abstract}

Keywords Periodic fundamental solution - Elliptic differential equation - Real analytic dependence $\cdot$ Helmholtz equation $\cdot$ Laplace equation

Mathematics Subject Classification $47 \mathrm{H} 30 \cdot 42 \mathrm{~B} 99 \cdot 31 \mathrm{~B} 10 \cdot 45 \mathrm{~A} 05 \cdot 35 \mathrm{~J} 25$

\begin{abstract}
The authors acknowledge the support of "Progetto di Ateneo: Singular perturbation problems for differential operators-CPDA120171/12"-University of Padova, and the support of "INdAM GNAMPA Project 2015-Un approccio funzionale analitico per problemi di perturbazione singolare e di omogeneizzazione." M. Lanza de Cristoforis acknowledges the support of the Grant EP/M013545/1: "Mathematical Analysis of Boundary-Domain Integral Equations for Nonlinear PDEs" from the EPSRC, UK. P. Musolino acknowledges the support of an "assegno di ricerca INdAM." P. Musolino has received funding from the European Union's Horizon 2020 research and innovation program under the Marie Skłodowska-Curie Grant Agreement No. 663830 and from the Welsh Government and Higher Education Funding Council for Wales through the Sêr Cymru National Research Network for Low Carbon, Energy and Environment.
\end{abstract}

$凶 \quad$ M. Lanza de Cristoforis mldc@math.unipd.it

1 Dipartimento di Matematica, Università degli Studi di Padova, Via Trieste 63, 35121 Padua, Italy

2 Department of Mathematics, Aberystwyth University, Ceredigion SY23 3BZ, Wales, UK 


\section{Introduction}

In this paper, we analyze analyticity properties of an analog of the periodic fundamental solution of an elliptic operator with constant coefficients jointly in the spatial variable and in the parameters which determine the size of the periodicity cell. We first introduce some notation. We fix once for all

$$
n \in \mathbb{N} \backslash\{0,1\} .
$$

Then we take

$$
\left.\left(q_{11}, \ldots, q_{n n}\right) \in\right] 0,+\infty\left[^{n},\right.
$$

and we introduce a periodicity cell

$$
\left.Q \equiv \Pi_{j=1}^{n}\right] 0, q_{j j}[.
$$

Then we denote by $q$ the diagonal matrix

$$
q \equiv\left(\begin{array}{cccc}
q_{11} & 0 & \ldots & 0 \\
0 & q_{22} & \ldots & 0 \\
\ldots & \ldots & \ldots & \ldots \\
0 & 0 & \ldots & q_{n n}
\end{array}\right)
$$

and by $m_{n}(Q)$ the $n$-dimensional measure of the fundamental cell $Q$. Clearly, $q \mathbb{Z}^{n} \equiv\{q z$ : $\left.z \in \mathbb{Z}^{n}\right\}$ is the set of vertices of a periodic subdivision of $\mathbb{R}^{n}$ corresponding to the fundamental cell $Q$, and accordingly, one can speak about $q$-periodic functions or distributions in $\mathbb{R}^{n}$. Next we introduce a family of differential operators. Let $N_{2}$ denote the number of multi-indexes $\alpha \in \mathbb{N}^{n}$ with $|\alpha| \leq 2$. For each $\mathbf{c} \equiv\left(c_{\alpha}\right)_{|\alpha| \leq 2} \in \mathbb{C}^{N_{2}}$, we set

$$
\mathbf{c}^{(2)} \equiv\left(c_{l j}^{(2)}\right)_{l, j=1, \ldots, n} \quad \mathbf{c}^{(1)} \equiv\left(c_{j}\right)_{j=1, \ldots, n}
$$

with $c_{l j}^{(2)} \equiv 2^{-1} c_{e_{l}+e_{j}}$ for $j \neq l, c_{j j}^{(2)} \equiv c_{e_{j}+e_{j}}$, and $c_{j} \equiv c_{e_{j}}$, where $\left\{e_{j}: j=1, \ldots, n\right\}$ is the canonical basis of $\mathbb{R}^{n}$. We note that the matrix $\mathbf{c}^{(2)}$ is symmetric. If $\mathbf{c} \in \mathbb{C}^{N_{2}}$, then we set

$$
P[\mathbf{c}, x] \equiv \sum_{|\alpha| \leq 2} c_{\alpha} x^{\alpha} \quad \forall x \in \mathbb{R}^{n} .
$$

We also set

$$
\mathcal{E} \equiv\left\{\mathbf{c} \equiv\left(c_{\alpha}\right)_{|\alpha| \leq 2} \in \mathbb{C}^{N_{2}}: \inf _{\xi \in \mathbb{R}^{n},|\xi|=1} \operatorname{Re}\left\{\sum_{|\alpha|=2} c_{\alpha} \xi^{\alpha}\right\}>0\right\} .
$$

Clearly, $\mathcal{E}$ coincides with the set of coefficients $\mathbf{c} \equiv\left(c_{\alpha}\right)_{|\alpha| \leq 2}$ such that the differential operator

$$
P[\mathbf{c}, D] \equiv \sum_{|\alpha| \leq 2} c_{\alpha} D^{\alpha}
$$

is strongly elliptic and has complex coefficients. As is well known, if $\mathbf{c} \in \mathcal{E}$, a $q$-periodic distribution $G$ is a $q$-periodic fundamental solution of $P[\mathbf{c}, D]$ provided that

$$
P[\mathbf{c}, D] G=\sum_{z \in \mathbb{Z}^{n}} \delta_{q z},
$$


where $\delta_{q z}$ denotes the Dirac measure with mass at $q z$, for all $z \in \mathbb{Z}^{n}$.

Unfortunately however, not all operators $P[\mathbf{c}, D]$ admit $q$-periodic fundamental solutions, not even in case $P[\mathbf{c}, D]$ is the Laplace operator.

Instead, if we denote by $E_{2 \pi i \xi}$, the function defined by

$$
E_{2 \pi i \xi}(x) \equiv e^{2 \pi i \xi \cdot x} \quad \forall x \in \mathbb{R}^{n},
$$

for all $\xi \in \mathbb{R}^{n}$ and if $\mathbf{c} \in \mathcal{E}$, then one can show that the set

$$
\mathbb{Z}(\mathbf{c}, q) \equiv\left\{z \in \mathbb{Z}^{n}: P\left[\mathbf{c}, 2 \pi i q^{-1} z\right]=0\right\}
$$

is finite and that the $q$-periodic distribution

$$
S_{\mathbf{c}, q} \equiv \sum_{z \in \mathbb{Z}^{n} \backslash \mathbb{Z}(\mathbf{c}, q)} \frac{1}{m_{n}(Q)} \frac{1}{P\left[\mathbf{c}, 2 \pi i q^{-1} z\right]} E_{2 \pi i q^{-1} z}
$$

satisfies the equality

$$
P[\mathbf{c}, D]\left(S_{\mathbf{c}, q}\right)=\sum_{z \in \mathbb{Z}^{n}} \delta_{q z}-\sum_{z \in \mathbb{Z}(\mathbf{c}, q)} \frac{1}{m_{n}(Q)} E_{2 \pi i q^{-1} z}
$$

(cf. e.g., Ammari and Kang [1, p. 53], [16, §3]). Now let $\mathbf{c} \in \mathcal{E}$ be fixed. We are interested into the analysis of perturbation problems for the kernel $S_{\mathbf{c}, q}$ and into the dependence of $S_{\mathbf{c}, q}$ upon $q$ and the spatial variable $x$, and we note that by perturbing $q$, the set $\mathbb{Z}(\mathbf{c}, q)$ is not stable. To circumvent such a difficulty, we fix a finite subset $\mathcal{Z}$ of $\mathbb{Z}^{n}$, and we consider those c and $q$ such that $\mathbb{Z}(\mathbf{c}, q) \subseteq \mathcal{Z}$. Then we note that

$$
S_{\mathbf{c}, q, \mathcal{Z}} \equiv \sum_{z \in \mathbb{Z}^{n} \backslash \mathcal{Z}} \frac{1}{m_{n}(Q)} \frac{1}{P\left[\mathbf{c}, 2 \pi i q^{-1} z\right]} E_{2 \pi i q^{-1} z}
$$

satisfies the equality

$$
P[\mathbf{c}, D]\left(S_{\mathbf{c}, q, \mathcal{Z}}\right)=\sum_{z \in \mathbb{Z}^{n}} \delta_{q z}-\sum_{z \in \mathcal{Z}} \frac{1}{m_{n}(Q)} E_{2 \pi i q^{-1} z} .
$$

Equality (3) can be considered as an effective substitute of equality (1), and we say that $S_{\mathbf{c}, q, \mathcal{Z}}$ is a $\mathcal{Z}$-analog of a $q$-periodic fundamental solution of $P[\mathbf{c}, D]$.

Clearly, the distribution $S_{\mathbf{c}, q, \mathcal{Z}}$ differs from $S_{\mathbf{c}, q}$ by an entire analytic function. Moreover, by interior elliptic regularity theory, both $S_{\mathbf{c}, q, \mathcal{Z}}$ and $S_{\mathbf{c}, q}$ are analytic in the open set $\mathbb{R}^{n} \backslash q \mathbb{Z}^{n}$.

Let $S_{\mathbf{c}}$ be a locally integrable real-valued function in $\mathbb{R}^{n}$ such that

$$
P[\mathbf{c}, D] S_{\mathbf{c}}=\delta_{0} \quad \text { in } \mathbb{R}^{n},
$$

in the sense of distributions. Then $S_{\mathbf{c}}$ is a fundamental solution for $P[\mathbf{c}, D]$, and the function $S_{\mathbf{c}, q, \mathcal{Z}}-S_{\mathbf{c}}$ can be extended to an analytic function in $\left(\mathbb{R}^{n} \backslash q \mathbb{Z}^{n}\right) \cup\{0\}$.

We denote such an extension of $S_{\mathbf{c}, q, \mathcal{Z}}-S_{\mathbf{c}}$ by the symbol $R_{\mathbf{c}, q, \mathcal{Z}, S_{\mathbf{c}}}$, and we say that $R_{\mathbf{c}, q, \mathcal{Z}, S_{\mathbf{c}}}$ is the regular part of $S_{\mathbf{c}, q, \mathcal{Z}}$ (with respect to $S_{\mathbf{c}}$ ). Obviously, $R_{\mathbf{c}, q, \mathcal{Z}, S_{\mathbf{c}}}$ is not a $q$-periodic function.

In this paper we are interested in various questions on the analyticity of $S_{\mathbf{c}, q, \mathcal{Z}}$ and $R_{\mathbf{c}, q, \mathcal{Z}, S_{\mathbf{c}}}$ in the variable $(q, x)$. Here the difficulty is that the series in (2) is known to converge only in the sense of distributions. We mention that Lin and Wang [20], Mityushev and Adler [24], and Mamode [22] have proved the validity of a constructive formula for a $q$-periodic analog of the fundamental solution for the Laplace operator in case $n=2$ via elliptic functions which would imply the analyticity of $S_{\mathbf{c}, q, \mathcal{Z}}$ and $R_{\mathbf{c}, q, \mathcal{Z}, S_{\mathbf{c}}}$ in the variable $(q, x)$. However, 
we are not aware of such formulas for $n \geq 3$ or for elliptic differential operators other than the Laplace operator.

We denote by $\mathbb{D}_{n}(\mathbb{R})$ the space of $n \times n$ diagonal matrices with real entries and by $\mathbb{D}_{n}^{+}(\mathbb{R})$ the set of elements of $\mathbb{D}_{n}(\mathbb{R})$ with diagonal entries in $] 0,+\infty[$.

We note that if we fix $s \in \mathbb{R}$ such that $s-2<-(n / 2)$, then $S_{\mathbf{c}, q, \mathcal{Z}}$ belongs to the Sobolev-Bessel potential space of $I$-periodic functions $H_{I}^{s}\left(\mathbb{R}^{n}\right)$, and we prove that the map from the set of $q$ in $\mathbb{D}_{n}^{+}(\mathbb{R})$ such that $\mathbb{Z}(\mathbf{c}, q) \subseteq \mathcal{Z}$ to $H_{I}^{s}\left(\mathbb{R}^{n}\right)$ which takes $q$ to $S_{\mathbf{c}, q, \mathcal{Z}} \circ q$ is real analytic (see Theorem 1). Here $I$ denotes the $n \times n$ identity matrix.

Then as an application we consider the Helmholtz operator $\Delta+\kappa^{2}$ for some $\kappa \in \mathbb{C}$, and we denote by $\mathbf{c}(\kappa)$, the element of $\mathcal{E}$ such that $P[\mathbf{c}(\kappa), D]=\Delta+\kappa^{2}$ (cf. 15-17). In this paper, we consider only the case in which $\kappa=0$ and $\mathbb{Z}(\mathbf{c}(0), q) \subseteq \mathcal{Z}=\{0\}$, and the case in which $\kappa \neq 0$ and $\mathbb{Z}(\mathbf{c}(\kappa), q) \subseteq \mathcal{Z}=\emptyset$, a case in which $-\kappa^{2}$ is not an eigenvalue for $\Delta$ in the space of $q$-periodic distributions in $\mathbb{R}^{n}$. Then we prove that if $\Omega$ is a bounded open subset of $\left.\mathbb{R}^{n} \backslash \mathbb{Z}^{n}, m \in \mathbb{N}, \alpha \in\right] 0,1[$, then the map which takes $q$ to the restriction to $\operatorname{cl} \Omega$ of the function

$$
S_{\mathbf{c}(\kappa), q, \mathcal{Z}} \circ q(x) \equiv S_{\mathbf{c}(\kappa), q, \mathcal{Z}}(q x) \quad \forall x \in \mathbb{R}^{n} \backslash \mathbb{Z}^{n},
$$

is real analytic from suitable subsets of $\mathbb{D}_{n}^{+}(\mathbb{R})$ to $C^{m, \alpha}(\operatorname{cl} \Omega)$ (see Theorem 3).

Then we prove an analyticity result for the regular part of $S_{\mathbf{c}(\kappa), q, \mathcal{Z}}$ in the Roumieu space $C_{\omega, \rho}^{0}(\operatorname{cl} \Omega)$ of real analytic functions in $\operatorname{cl} \Omega$ in case $\operatorname{cl} \Omega \subseteq\left(\mathbb{R}^{n} \backslash \mathbb{Z}^{n}\right) \cup\{0\}$ (cf. (5) and see Theorem 4).

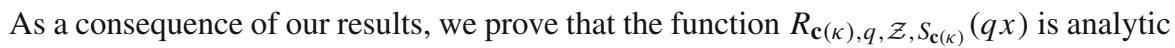
in the variable $(q, x)$ (cf. Theorem 5), and that $S_{\mathbf{c}(\kappa), q, \mathcal{Z}}(q x)$ is analytic in the variable $(q, x)$ (cf. Theorem 7). In particular, we can deduce that the sum of the series

$$
-\sum_{z \in \mathbb{Z}^{n} \backslash\{0\}} \frac{1}{m_{n}(Q) 4 \pi^{2}\left|q^{-1} z\right|^{2}} e^{2 \pi i\left(q^{-1} z\right) \cdot x},
$$

which converges in the sense of distributions to an analog of the $q$-periodic fundamental solution of the Laplace operator defines an analytic function of $(q, x) \in \mathbb{D}_{n}^{+}(\mathbb{R}) \times \mathbb{R}^{n}$ such that $q^{-1} x \notin \mathbb{Z}^{n}$, i.e., jointly in the variables $q$ and $x$ (see Example 1 at the end of the paper). For a corresponding example for the Helmholtz operator, see Example 2 at the end of the paper.

A central tool in periodic potential theory is represented by analogs of the periodic fundamental solution. As an example, Ammari et al. [3] have exploited an integral equation method to solve a periodic linear transmission problem for the Laplace equation and to derive effective properties of composite materials. Such an approach has been successfully exploited also for the study of the effective parameters of elastic composites in Ammari et al. [2] (see also Ammari and Kang [1]).

An approach based on potential theory has been useful also for the analysis of nonlinear periodic problems. For example, in [18] a quasilinear heat transmission problem has been investigated by means of integral equations, whereas in [10] such an analysis has been performed for a nonlinear traction problem.

We also mention that Arens et al. [4], Berman and Greengard [5], Tornberg and Greengard [26] have investigated the problem of actually computing the sum of series as that of (4).

The analysis of the present paper is motivated by the application of the potential theoretic method to boundary value problems corresponding to anisotropic periodic problems in which the sizes $q_{11}, \ldots, q_{n n}$ of the periodic cell are subject to perturbation. Indeed, if one wants to apply periodic potential theory to study the dependence of the solution of a periodic 
boundary value problem upon the parameters $q_{11}, \ldots, q_{n n}$ which determine the anisotropy of the problem, then one faces the problem to study the corresponding dependence for the fundamental solution on which the potentials are based. In particular, an analyticity result upon the parameters $q_{11}, \ldots, q_{n n}$ allows to justify representation formulas for the solutions or for functionals related to the solutions in terms of power series in $q_{11}, \ldots, q_{n n}$ and therefore also polynomial asymptotic expansions of any desired degree with precise estimates on the remainder.

This paper continues the work of the authors and collaborators on the study of the behavior of the fundamental solution of an elliptic partial differential operator upon perturbation of the coefficients. For example, in [7], Dalla Riva has constructed a family of fundamental solutions for elliptic partial differential operators with real constant coefficients, where the elements of such a family are expressed by means of real analytic functions of the coefficients of the operators and of the spatial variable. Then a corresponding result for elliptic partial differential operators with quaternion constant coefficients has been shown in [9].

The paper is organized as follows. In Sect. 2 we introduce some preliminaries, in particular on periodic distributions. In Sect. 3, we prove the analyticity result on $S_{\mathbf{c}, q, \mathcal{Z}} \circ q$ upon $q \in \mathbb{D}_{n}^{+}(\mathbb{R})$ in Sobolev-Bessel spaces. In Sect. 4 we compute all the differentials of a map related to $S_{\mathbf{c}, q, \mathcal{Z}} \circ q$, which we need in the sequel.

In Sect. 5 we consider the Helmholtz operator. We first prove the above-mentioned analyticity result for $S_{\mathbf{c}(\kappa), q, \mathcal{Z}} \circ q_{\mid \mathrm{cl} \Omega}$, with values in $C^{m, \alpha}(\mathrm{cl} \Omega)$.

Then we prove the analyticity of $R_{\mathbf{c}(\kappa), q, \mathcal{Z}, S_{\mathbf{c}(\kappa)}} \circ q_{\mid \mathrm{cl} \Omega}$ upon $q$ in Roumieu spaces and the joint analyticity of the function $R_{\mathbf{c}(\kappa), q, \mathcal{Z}, S_{\mathbf{c}}}(q x)$ upon $(q, x)$. Finally, we prove the analyticity $S_{\mathbf{c}(\kappa), q, \mathcal{Z}} \circ q_{\mid \mathrm{cl} \Omega}$ upon $q$ in Roumieu spaces and the joint analyticity of the function $S_{\mathbf{c}(\kappa), q, \mathcal{Z}}(q x)$ upon $(q, x)$.

\section{Preliminaries and notation}

We denote the norm on a normed space $\mathcal{X}$ by $\|\cdot\| \mathcal{X}$. Let $\mathcal{X}$ and $\mathcal{Y}$ be normed spaces. We endow the space $\mathcal{X} \times \mathcal{Y}$ with the norm defined by $\|(x, y)\| \mathcal{X} \times \mathcal{Y} \equiv\|x\|_{\mathcal{X}}+\|y\|_{\mathcal{Y}}$ for all $(x, y) \in \mathcal{X} \times \mathcal{Y}$, while we use the Euclidean norm for $\mathbb{R}^{n}$. The symbol $\mathbb{N}$ denotes the set of natural numbers including $0 . \mathcal{L}(\mathcal{X}, \mathcal{Y})$ denotes the space of linear and continuous operators from $\mathcal{X}$ to $\mathcal{Y}$. Let $\mathbb{E} \subseteq \mathbb{R}^{n}$. Then cl $\mathbb{E}$ denotes the closure of $\mathbb{E}$, and $\partial \mathbb{E}$ denotes the boundary of $\mathbb{E}$. For all $R>0, x \in \mathbb{R}^{n}, x_{j}$ denotes the $j$-th coordinate of $x,|x|$ denotes the Euclidean modulus of $x$ in $\mathbb{R}^{n}$, and $\mathbb{B}_{n}(x, R)$ denotes the ball $\left\{y \in \mathbb{R}^{n}:|x-y|<R\right\}$. A dot "." denotes the inner product in $\mathbb{R}^{n}$, or the matrix product between matrices. Let $\Omega$ be an open subset of $\mathbb{R}^{n}$. The space of $m$ times continuously differentiable complex-valued functions on $\Omega$ is denoted by $C^{m}(\Omega, \mathbb{R})$, or more simply by $C^{m}(\Omega)$. Let $r \in \mathbb{N} \backslash\{0\}$. Let $f \in\left(C^{m}(\Omega)\right)^{r}$. The $s$-th component of $f$ is denoted $f_{s}$, and $D f$ denotes the Jacobian matrix $\left(\frac{\partial f_{s}}{\partial x_{l}}\right)_{\substack{s=1, \ldots, r \\ l=1, \ldots, n}}$. Let $\eta \equiv\left(\eta_{1}, \ldots, \eta_{n}\right) \in \mathbb{N}^{n},|\eta| \equiv \eta_{1}+\cdots+\eta_{n}$. Then $D^{\eta} f$ denotes $\frac{\partial^{|\eta|} f}{\partial x_{1}^{\eta_{1}} \ldots \partial x_{n}^{\eta_{n}}}$. We denote by $\mathcal{D}\left(\mathbb{R}^{n}\right)$ the space of functions of class $C^{\infty}\left(\mathbb{R}^{n}\right)$ with compact support. The subspace of $C^{m}(\Omega)$ of those functions $f$ whose derivatives $D^{\eta} f$ of order $|\eta| \leq m$ can be extended with continuity to $\operatorname{cl} \Omega$ is denoted $C^{m}(\operatorname{cl} \Omega)$. The subspace of $C^{m}(\operatorname{cl} \Omega)$ whose functions have $m$-th-order derivatives that are Hölder continuous with exponent $\alpha \in] 0,1]$ is denoted $C^{m, \alpha}(\mathrm{cl} \Omega)$ (cf. e.g., Gilbarg and Trudinger [13]). Let $\mathbb{E} \subseteq \mathbb{R}^{r}$. Then $C^{m, \alpha}(\mathrm{cl} \Omega, \mathbb{E})$ denotes $\left\{f \in\left(C^{m, \alpha}(\operatorname{cl} \Omega)\right)^{r}: f(\operatorname{cl} \Omega) \subseteq \mathbb{E}\right\}$. 
We say that a bounded open subset $\Omega$ of $\mathbb{R}^{n}$ is of class $C^{m}$ or of class $C^{m, \alpha}$, if $\operatorname{cl} \Omega$ is a manifold with boundary imbedded in $\mathbb{R}^{n}$ of class $C^{m}$ or $C^{m, \alpha}$, respectively (cf. e.g., Gilbarg and Trudinger [13, §6.2]). For standard properties of functions in Schauder spaces both on $\operatorname{cl} \Omega$ and on $\partial \Omega$, we refer the reader to Gilbarg and Trudinger [13] (see also [15, §2, Lem. 3.1, 4.26, Thm. 4.28], [19, §2]).

We denote by $\mathrm{d} \sigma$ the area element of a manifold $M$ imbedded into $\mathbb{R}^{n}$. We retain the standard notation for the Lebesgue space $L^{p}(M)$ of $p$-summable functions. We note that throughout the paper "analytic" means always "real analytic." For the definition and properties of analytic operators, we refer to Deimling [11, §15].

Next, we turn to introduce the Roumieu classes. For all bounded open subsets $\Omega$ of $\mathbb{R}^{n}$ and $\rho>0$, we set

$$
C_{\omega, \rho}^{0}(\operatorname{cl} \Omega) \equiv\left\{u \in C^{\infty}(\operatorname{cl} \Omega): \sup _{\beta \in \mathbb{N}^{n}} \frac{\rho^{|\beta|}}{|\beta| !}\left\|D^{\beta} u\right\|_{C^{0}(\mathrm{cl} \Omega)}<+\infty\right\},
$$

and

$$
\|u\|_{C_{\omega, \rho}^{0}(\operatorname{cl} \Omega)} \equiv \sup _{\beta \in \mathbb{N}^{n}} \frac{\rho^{|\beta|}}{|\beta| !}\left\|D^{\beta} u\right\|_{C^{0}(\mathrm{cl} \Omega)} \quad \forall u \in C_{\omega, \rho}^{0}(\operatorname{cl} \Omega),
$$

where $|\beta| \equiv \beta_{1}+\cdots+\beta_{n}$ for all $\beta \equiv\left(\beta_{1}, \ldots, \beta_{n}\right) \in \mathbb{N}^{n}$. As is well known, the Roumieu class $\left(C_{\omega, \rho}^{0}(\operatorname{cl} \Omega),\|\cdot\|_{C_{\omega, \rho}^{0}(\operatorname{cl} \Omega)}\right)$ is a Banach space.

We denote by $\mathcal{S}\left(\mathbb{R}^{n}\right)$ the Schwartz space of rapidly decreasing functions, and by $\mathcal{S}^{\prime}\left(\mathbb{R}^{n}\right)$ the space of tempered distributions in $\mathbb{R}^{n}$, and by $\mathcal{S}_{I}^{\prime}\left(\mathbb{R}^{n}\right)$ the subspace of $\mathcal{S}^{\prime}\left(\mathbb{R}^{n}\right)$ of the $I$-periodic elements of $\mathcal{S}^{\prime}\left(\mathbb{R}^{n}\right)$, i.e., of the tempered distributions which are periodic with respect to the fundamental cell $] 0,1\left[{ }^{n}\right.$. If $f$ is a complex-valued integrable function in $\mathbb{R}^{n}$, then we define the Fourier transform of $f$ as follows

$$
\hat{f}(y) \equiv(2 \pi)^{-n / 2} \int_{\mathbb{R}^{n}} e^{-i y \cdot x} f(x) \mathrm{d} x \quad \forall y \in \mathbb{R}^{n},
$$

and we still use the symbol "^" to denote the corresponding Fourier transform in the space of tempered distributions. Next we introduce the following characterization of $\mathcal{S}_{I}^{\prime}\left(\mathbb{R}^{n}\right)$ of Triebel [27] (see also Schmeisser and Triebel [25, 3.2.3]).

Proposition 1 If $\left\{a_{z}\right\}_{z \in \mathbb{Z}^{n}}$ is a family of complex numbers such that there exists $m \in \mathbb{N}$ such that

$$
\sup _{z \in \mathbb{Z}^{n}} \frac{\left|a_{z}\right|}{\left(1+|z|^{2}\right)^{m / 2}}<+\infty
$$

then the Fourier series

$$
\sum_{z \in \mathbb{Z}^{n}} a_{z} e^{i 2 \pi z \cdot x}
$$

converges in $\mathcal{S}^{\prime}\left(\mathbb{R}^{n}\right)$ endowed with the weak ${ }^{*}$-topology to an element of $\mathcal{S}_{I}^{\prime}\left(\mathbb{R}^{n}\right)$.

Conversely, if $u \in \mathcal{S}_{I}^{\prime}\left(\mathbb{R}^{n}\right)$, then there exists a unique family $\left\{a_{z}(u)\right\}_{z \in \mathbb{Z}^{n}}$ in $\mathbb{C}$ which satisfies condition (6) for some $m \in \mathbb{N}$ and such that $u$ equals the sum of the Fourier series in (7) with $a_{z}$ replaced by $a_{z}(u)$. Moreover,

$$
a_{k}(u)=(2 \pi)^{-n / 2}<u,[\varphi(\cdot-2 \pi k)]^{\wedge}>\quad \forall k \in \mathbb{Z}^{n},
$$

for all $\varphi \in \mathcal{D}\left(\mathbb{R}^{n}\right)$ which have support contained in the ball $\mathbb{B}_{n}(0,2 \pi)$ and such that $\varphi(0)=1$. 
We also note that the map from $\mathcal{S}_{I}^{\prime}\left(\mathbb{R}^{n}\right)$ endowed with the weak*-topology to $\mathbb{C}$ which takes $u$ to $a_{k}(u)$ is linear and continuous for all $k \in \mathbb{Z}^{n}$.

If $s \in \mathbb{R}$, then we denote by $H^{s}\left(\mathbb{R}^{n}\right)$ the Sobolev-Bessel space of tempered distributions $u$ such that $\left(1+|y|^{2}\right)^{s / 2} \hat{u}(y)$ belongs to $L^{2}\left(\mathbb{R}^{n}\right)$, and we set

$$
\|u\|_{H^{s}\left(\mathbb{R}^{n}\right)} \equiv\left\|\left(1+|y|^{2}\right)^{s / 2} \hat{u}(y)\right\|_{L^{2}\left(\mathbb{R}^{n}\right)} \quad \forall u \in H^{s}\left(\mathbb{R}^{n}\right) .
$$

It is well known that $\left(H^{s}\left(\mathbb{R}^{n}\right),\|\cdot\|_{H^{s}\left(\mathbb{R}^{n}\right)}\right)$ is a Banach space. Then we set

$$
H_{\mathrm{loc}}^{s}\left(\mathbb{R}^{n}\right) \equiv\left\{u \in \mathcal{S}^{\prime}\left(\mathbb{R}^{n}\right): u \varphi \in H^{s}\left(\mathbb{R}^{n}\right) \forall \varphi \in \mathcal{D}\left(\mathbb{R}^{n}\right)\right\},
$$

and

$$
\|u\|_{H_{\mathrm{loc}}^{s}\left(\mathbb{R}^{n}\right), \varphi} \equiv\|u \varphi\|_{H^{s}\left(\mathbb{R}^{n}\right)} \quad \forall u \in H_{\mathrm{loc}}^{s}\left(\mathbb{R}^{n}\right),
$$

for all $\varphi \in \mathcal{D}\left(\mathbb{R}^{n}\right)$. Then it is well known that $H_{\text {loc }}^{s}\left(\mathbb{R}^{n}\right)$ endowed with the family of seminorms $\Phi \equiv\left\{\|\cdot\|_{H_{\mathrm{loc}}^{s}\left(\mathbb{R}^{n}\right), \varphi}: \varphi \in \mathcal{D}\left(\mathbb{R}^{n}\right)\right\}$ is a Fréchet space. Next we introduce the space

$$
H_{I}^{s}\left(\mathbb{R}^{n}\right) \equiv H_{\text {loc }}^{s}\left(\mathbb{R}^{n}\right) \cap \mathcal{S}_{I}^{\prime}\left(\mathbb{R}^{n}\right) .
$$

Clearly, $H_{I}^{S}\left(\mathbb{R}^{n}\right)$ is a closed subspace of the Fréchet space $H_{\mathrm{loc}}^{s}\left(\mathbb{R}^{n}\right)$ and is accordingly a Fréchet space. Next we fix an arbitrary $\eta \in \mathcal{D}\left(\mathbb{R}^{n}\right)$ such that there exists an open neighborhood $U$ of $[0,1]^{n}$ such that

$$
\eta(x)=1 \quad \forall x \in U .
$$

Since the tempered distributions of $H_{I}^{s}\left(\mathbb{R}^{n}\right)$ are $I$-periodic, one can easily verify that $\|\cdot\|_{H_{\mathrm{loc}}^{s}\left(\mathbb{R}^{n}\right), \eta}$ is actually a norm on $H_{I}^{s}\left(\mathbb{R}^{n}\right)$. Since $\|\cdot\|_{H_{\mathrm{loc}}^{s}\left(\mathbb{R}^{n}\right), \eta}$ belongs to $\Phi$ and $H_{I}^{s}\left(\mathbb{R}^{n}\right)$ is a Fréchet space, we already know that $\left(H_{I}^{s}\left(\mathbb{R}^{n}\right),\|\cdot\|_{H_{\mathrm{loc}}^{s}}\left(\mathbb{R}^{n}\right), \eta\right)$ is complete. Then the open mapping theorem in Fréchet spaces implies that the continuous identity map from $\left(H_{I}^{s}\left(\mathbb{R}^{n}\right), \Phi\right)$ to $\left(H_{I}^{s}\left(\mathbb{R}^{n}\right),\|\cdot\|_{H_{\text {loc }}^{s}\left(\mathbb{R}^{n}\right), \eta}\right)$ is actually a homeomorphism and that accordingly $\|\cdot\|_{H_{\mathrm{loc}}^{s}}\left(\mathbb{R}^{n}\right), \eta$ generates the topology of the Fréchet space $\left(H_{I}^{s}\left(\mathbb{R}^{n}\right), \Phi\right)$, no matter how we choose $\eta \in \mathcal{D}\left(\mathbb{R}^{n}\right)$ as in (9).

Now let $\Omega$ be an open subset of $\mathbb{R}^{n}$, then we denote by $H^{s}(\Omega)$ the set of restrictions to $\Omega$ of the tempered distributions of $H^{s}\left(\mathbb{R}^{n}\right)$, and we set

$$
\|u\|_{H^{s}(\Omega)} \equiv \inf \left\{\|v\|_{H^{s}\left(\mathbb{R}^{n}\right)}: v \in H^{s}\left(\mathbb{R}^{n}\right), v_{\mid \Omega}=u\right\} \quad \forall u \in H^{s}(\Omega) .
$$

It is well known that $\left(H^{s}(\Omega),\|\cdot\|_{H^{s}(\Omega)}\right)$ is a Banach space. We note that such a definition of $H^{S}(\Omega)$ coincides with other "intrinsic" definitions of $H^{S}(\Omega)$ only in case $\Omega$ satisfies some regularity assumption.

If $\Omega$ is a bounded open subset of $\mathbb{R}^{n}$, and if we choose $\eta \in \mathcal{D}\left(\mathbb{R}^{n}\right)$ as in (9) such that $\eta$ equals one in a neighborhood of $\operatorname{cl} \Omega$, then the definition of the norm in $H^{s}(\Omega)$ implies that the restriction map is linear and continuous from $H_{I}^{s}\left(\mathbb{R}^{n}\right)$ to $H^{s}(\Omega)$ and that

$$
\left\|u_{\mid \Omega}\right\|_{H^{s}(\Omega)} \leq\|u\|_{H_{\mathrm{loc}}^{s}\left(\mathbb{R}^{n}\right), \eta} \quad \forall u \in H_{I}^{s}\left(\mathbb{R}^{n}\right) .
$$

If $\mathbf{c} \in \mathcal{E}, q \in \mathbb{D}_{n}^{+}(\mathbb{R})$, then we set

$$
L_{\mathbf{c}, q}[u] \equiv \sum_{|\alpha| \leq 2}\left(q^{-1}\right)^{\alpha} c_{\alpha} D^{\alpha} u \quad \forall u \in \mathcal{S}^{\prime}\left(\mathbb{R}^{n}\right),
$$

where $\left(q^{-1}\right)^{\alpha} \equiv q_{11}^{-\alpha_{1}} \ldots q_{n n}^{-\alpha_{n}}$ for all $\alpha \in \mathbb{N}^{n}$, and we have the following.

Lemma 1 Let $\mathbf{c} \in \mathcal{E}$. Let $S_{\mathbf{c}}$ be a fundamental solution for $P[\mathbf{c}, D]$. Let $q \in \mathbb{D}_{n}^{+}(\mathbb{R})$. Let $\mathcal{Z}$ be a finite subset of $\mathbb{Z}^{n}$ such that $\mathbb{Z}(\mathbf{c}, q) \subseteq \mathcal{Z}$. Then the following statements hold. 
(i)

$$
L_{\mathbf{c}, q}\left[(\operatorname{det} q) S_{\mathbf{c}, q, \mathcal{Z}} \circ q\right]=-\sum_{z \in \mathcal{Z}} E_{2 \pi i z} \text { in } \mathbb{R}^{n} \backslash \mathbb{Z}^{n},
$$

and

$$
L_{\mathbf{c}, q}\left[(\operatorname{det} q) R_{\mathbf{c}, q, \mathcal{Z}, S_{\mathbf{c}}} \circ q\right]=-\sum_{z \in \mathcal{Z}} E_{2 \pi i z} \text { in }\left(\mathbb{R}^{n} \backslash \mathbb{Z}^{n}\right) \cup\{0\} .
$$

(ii) $L_{\mathbf{c}, q}\left[(\operatorname{det} q) S_{\mathbf{c}, q, \mathcal{Z}} \circ q\right]=\sum_{z \in \mathbb{Z}^{n}} \delta_{z}-\sum_{z \in \mathcal{Z}} E_{2 \pi i z}$ in $\mathcal{S}^{\prime}\left(\mathbb{R}^{n}\right)$.

(iii) $a_{k}\left(\sum_{z \in \mathbb{Z}^{n}} \delta_{z}-\sum_{z \in \mathcal{Z}} E_{2 \pi i z}\right)=0$ for all $k \in \mathcal{Z}(c f .8)$.

Proof The first equality in (i) follows by the chain rule. Since $L_{\mathbf{c}, q}\left[(\operatorname{det} q) S_{\mathbf{c}} \circ q\right]=0$ in $\mathbb{R}^{n} \backslash\{0\}$, the second equality in statement (i) holds true in $\mathbb{R}^{n} \backslash \mathbb{Z}^{n}$. Since $R_{\mathbf{c}, q, \mathcal{Z}, S_{\mathbf{c}}}$ can be continued analytically in a neighborhood of 0 , the second equality in statement (i) holds true in $\left(\mathbb{R}^{n} \backslash \mathbb{Z}^{n}\right) \cup\{0\}$. We now prove statement (ii). The equality of statement (ii) holds if and only if

$$
\begin{gathered}
\sum_{|\alpha| \leq 2}\left(-q^{-1}\right)^{\alpha} c_{\alpha} \int_{\mathbb{R}^{n}}(\operatorname{det} q) S_{\mathbf{c}, q, \mathcal{Z}}(q \xi) D^{\alpha} \psi(\xi) \mathrm{d} \xi \\
=\sum_{z \in \mathbb{Z}^{n}} \psi\left(q^{-1} q z\right)-\sum_{z \in \mathcal{Z}} \int_{\mathbb{R}^{n}} \psi(\xi) e^{i 2 \pi z \cdot \xi} \mathrm{d} \xi,
\end{gathered}
$$

for all $\psi \in \mathcal{S}\left(\mathbb{R}^{n}\right)$. By setting $x=q \xi$, we rewrite such an equality as

$$
\begin{aligned}
\int_{\mathbb{R}^{n}} S_{\mathbf{c}, q, \mathcal{Z}}(x) \sum_{|\alpha| \leq 2}(-1)^{|\alpha|} c_{\alpha} D^{\alpha}\left(\psi\left(q^{-1} x\right)\right) \mathrm{d} x \\
\quad=\sum_{z \in \mathbb{Z}^{n}}<\delta_{q z}, \psi\left(q^{-1} \cdot\right)>-\sum_{z \in \mathcal{Z}} \int_{\mathbb{R}^{n}} \psi\left(q^{-1} x\right) e^{i 2 \pi q^{-1} z \cdot x} \mathrm{~d} x(\operatorname{det} q)^{-1},
\end{aligned}
$$

for all $\psi \in \mathcal{S}\left(\mathbb{R}^{n}\right)$. Now such an equality is certainly satisfied. Indeed, $\psi\left(q^{-1} \cdot\right)$ belongs to $\mathcal{S}\left(\mathbb{R}^{n}\right)$ and

$$
P[\mathbf{c}, D] S_{\mathbf{c}, q, \mathcal{Z}}=\sum_{z \in \mathbb{Z}^{n}} \delta_{q z}-\sum_{z \in \mathcal{Z}} \frac{1}{m_{n}(Q)} E_{2 \pi i q^{-1} z} \quad \text { in } \mathcal{S}^{\prime}\left(\mathbb{R}^{n}\right) .
$$

Finally, to prove statement (iii), we note that the Poisson summation formula implies that

$$
a_{k}\left(\sum_{z \in \mathbb{Z}^{n}} \delta_{z}-\sum_{z \in \mathcal{Z}} E_{2 \pi i z}\right)=a_{k}\left(\sum_{z \in \mathbb{Z}^{n}} E_{2 \pi i z}-\sum_{z \in \mathcal{Z}} E_{2 \pi i z}\right)=0,
$$

for all $k \in \mathcal{Z}$.

Then we find convenient to introduce the following notation. Let $\mathcal{Z}$ be a finite subset of $\mathbb{Z}^{n}$. For each $s \in \mathbb{R}$, we set

$$
H_{I, \mathcal{Z}}^{s}\left(\mathbb{R}^{n}\right) \equiv\left\{u \in H_{I}^{s}\left(\mathbb{R}^{n}\right): a_{k}(u)=0 \quad \forall k \in \mathcal{Z}\right\}
$$

[cf. (8)]. By the continuity of the functionals $a_{k}(\cdot)$ of $(8)$ on $\mathcal{S}_{I}^{\prime}\left(\mathbb{R}^{n}\right)$, the space $H_{I, \mathcal{Z}}^{s}\left(\mathbb{R}^{n}\right)$ is closed in $H_{I}^{S}\left(\mathbb{R}^{n}\right)$, and it is accordingly a Banach space. Then we need the following result on the operator $L_{\mathbf{c}, q}$ in the space $\mathcal{S}_{I}^{\prime}\left(\mathbb{R}^{n}\right)$ of $I$-periodic tempered distributions. 
Proposition 2 Let $\mathbf{c} \in \mathcal{E}$. Let $q \in \mathbb{D}_{n}^{+}(\mathbb{R})$. Let $\mathcal{Z}$ be a finite subset of $\mathbb{Z}^{n}$ such that $\mathbb{Z}(\mathbf{c}, q) \subseteq$ $\mathcal{Z}$. Then the following statements hold.

(i) Let $u \in \mathcal{S}_{I}^{\prime}\left(\mathbb{R}^{n}\right)$. Then $L_{\mathbf{c}, q}[u]=0$ if and only if $u$ belongs to the complex vector space generated by $\left\{E_{2 \pi i k}: k \in \mathbb{Z}(\mathbf{c}, q)\right\}$.

(ii) Let $f \in \mathcal{S}_{I}^{\prime}\left(\mathbb{R}^{n}\right)$. Then there exists $u \in \mathcal{S}_{I}^{\prime}\left(\mathbb{R}^{n}\right)$ such that $f=L_{\mathbf{c}, q}[u]$ if and only if $a_{k}(f)=0$ for all $k \in \mathbb{Z}(\mathbf{c}, q)\left(c f\right.$. 8). Moreover, if $a_{k}(f)=0$ for all $k \in \mathcal{Z}$ we can choose $u$ so that $a_{k}(u)=0$ for all $k \in \mathcal{Z}$.

(iii) Let $s \in \mathbb{R}$. Then the operator $L_{\mathbf{c}, q}$ maps $H_{I}^{s}\left(\mathbb{R}^{n}\right)$ onto the subspace $H_{I, \mathbb{Z}(\mathbf{c}, q)}^{s-2}\left(\mathbb{R}^{n}\right)$ of $H_{I}^{s-2}\left(\mathbb{R}^{n}\right)$, and restricts a linear homeomorphism from the space $H_{I, \mathcal{Z}}^{s}\left(\mathbb{R}^{n}\right)$ onto $H_{I, \mathcal{Z}}^{s-2}\left(\mathbb{R}^{n}\right)$.

Proof (i) The sufficiency of the condition follows by the chain rule and by the definition of $\mathbb{Z}(\mathbf{c}, q)$. To prove necessity, we note that equality $L_{\mathbf{c}, q}[u]=0$ implies that

$$
P[\mathbf{c}, D]\left(u\left(q^{-1} \cdot\right)\right)=0 .
$$

On the other hand, by Proposition 1 there exists a unique family $\left\{a_{z}(u)\right\}_{z \in \mathbb{Z}^{n}}$ in $\mathbb{C}$ as in (6) such that

$$
u\left(q^{-1} \cdot\right)=\sum_{z \in \mathbb{Z}^{n}} a_{z}(u) E_{2 \pi i q^{-1} z} \quad \text { in } \mathcal{S}^{\prime}\left(\mathbb{R}^{n}\right)
$$

Accordingly,

$$
0=P[\mathbf{c}, D]\left(u\left(q^{-1} \cdot\right)\right)=\sum_{z \in \mathbb{Z}^{n}} a_{z}(u) P\left[\mathbf{c}, 2 \pi i q^{-1} z\right] E_{2 \pi i q^{-1} z}
$$

and so

$$
a_{z}(u) P\left[\mathbf{c}, 2 \pi i q^{-1} z\right]=0 \quad \forall z \in \mathbb{Z}^{n} .
$$

Thus, $a_{z}(u)=0$ for all $z \in \mathbb{Z}^{n} \backslash \mathbb{Z}(\mathbf{c}, q)$. Hence, the validity of statement (i) follows.

(ii) We first assume that $u$ exists. Then Proposition 1 implies that there exist $m \in \mathbb{N}$ and a family of complex numbers $\left\{b_{z}\right\}_{z \in \mathbb{Z}^{n}}$ satisfying condition (6) and equality $u=$ $\sum_{z \in \mathbb{Z}^{n}} b_{z} e^{i 2 \pi z \cdot x}$ in $\mathcal{S}^{\prime}\left(\mathbb{R}^{n}\right)$ endowed with the weak ${ }^{*}$-topology. Then by applying $L_{\mathbf{c}, q}$, we obtain

$$
L_{\mathbf{c}, q}[u]=\sum_{z \in \mathbb{Z}^{n} \backslash \mathbb{Z}(\mathbf{c}, q)}\left(P\left[\mathbf{c}, 2 \pi i q^{-1} z\right]\right) b_{z} e^{i 2 \pi z \cdot x}
$$

and accordingly $a_{k}\left(L_{\mathbf{c}, q}[u]\right)=0$ for all $k \in \mathbb{Z}(\mathbf{c}, q)$.

Next we assume that $a_{k}(f)=0$ for all $k \in \mathcal{Z}$ and we show the existence of $u$. By Proposition 1 there exist $m \in \mathbb{N}$ and a family of complex numbers $\left\{a_{z}\right\}_{z \in \mathbb{Z}^{n}}$ satisfying condition (6) and equality $f=\sum_{z \in \mathbb{Z}^{n}} a_{z} e^{i 2 \pi z \cdot x}$ in $\mathcal{S}^{\prime}\left(\mathbb{R}^{n}\right)$ endowed with the weak ${ }^{*}$-topology. Since $a_{k}(f)=0$ for all $k \in \mathcal{Z}$, we have $a_{k}=0$ for all $k \in \mathcal{Z}$. Then we set

$$
b_{k} \equiv 0 \quad \forall k \in \mathcal{Z}, \quad b_{z} \equiv \frac{a_{z}}{P\left[\mathbf{c}, 2 \pi i q^{-1} z\right]} \quad \forall z \in \mathbb{Z}^{n} \backslash \mathcal{Z} .
$$

Clearly, $\left\{b_{z}\right\}_{z \in \mathbb{Z}^{n}}$ satisfies condition (6) with $m$ replaced by $m-2$, and accordingly the series $\sum_{z \in \mathbb{Z}^{n}} b_{z} e^{i 2 \pi z \cdot x}$ converges in the weak ${ }^{*}$-topology and defines a $I$-periodic element $u$ of $\mathcal{S}^{\prime}\left(\mathbb{R}^{n}\right)$. Moreover, $a_{k}(u)=0$ for all $k \in \mathcal{Z}$. By definition of the coefficients $\left\{b_{z}\right\}_{z \in \mathbb{Z}^{n}}$, we have $L_{\mathbf{c}, q}[u]=f$. 
(iii) Since $H_{I}^{s}\left(\mathbb{R}^{n}\right)=H_{\mathrm{loc}}^{s}\left(\mathbb{R}^{n}\right) \cap \mathcal{S}_{I}^{\prime}\left(\mathbb{R}^{n}\right)$, the second-order differential operator $L_{\mathbf{c}, q}$ is linear and continuous from $H_{I}^{s}\left(\mathbb{R}^{n}\right)$ to $H_{I}^{s-2}\left(\mathbb{R}^{n}\right)$. By (ii), $L_{\mathbf{c}, q}$ maps $H_{I}^{s}\left(\mathbb{R}^{n}\right)$ into $H_{I, \mathbb{Z}(\mathbf{c}, q)}^{s-2}\left(\mathbb{R}^{n}\right)$. On the other hand if $f \in H_{I}^{s-2}\left(\mathbb{R}^{n}\right)$ and $a_{k}(f)=0$ for all $k \in \mathbb{Z}(\mathbf{c}, q)$, then statement (ii) ensures the existence of $u \in \mathcal{S}_{I}^{\prime}\left(\mathbb{R}^{n}\right)$ such that $f=L_{\mathbf{c}, q}[u]$ and $a_{k}(u)=0$ for all $k \in \mathbb{Z}(\mathbf{c}, q)$. Since $f \in H_{\text {loc }}^{s-2}\left(\mathbb{R}^{n}\right)$, then classical elliptic regularity theory ensures that $u \in H_{\mathrm{loc}}^{s}\left(\mathbb{R}^{n}\right)$ (cf. e.g., Folland [12, (6.33), p. 214]). Thus we conclude that $u \in H_{\mathrm{loc}}^{s}\left(\mathbb{R}^{n}\right) \cap \mathcal{S}_{I}^{\prime}\left(\mathbb{R}^{n}\right)=H_{I}^{s}\left(\mathbb{R}^{n}\right)$. Similarly, (ii) implies that $L_{\mathbf{c}, q}$ maps $H_{I, \mathcal{Z}}^{s}\left(\mathbb{R}^{n}\right)$ onto $H_{I, \mathcal{Z}}^{s-2}\left(\mathbb{R}^{n}\right)$. If $u \in H_{I}^{s}\left(\mathbb{R}^{n}\right)$, and $L_{\mathbf{c}, q}[u]=0$, and $a_{k}(u)=0$ for all $k \in \mathcal{Z}$, then point (i) ensures that $u$ belongs to the complex vector space generated by $\left\{E_{2 \pi i k}: k \in \mathbb{Z}(\mathbf{c}, q)\right\}$ and thus condition $a_{k}(u)=0$ for all $k \in \mathcal{Z}$ ensures that $u=0$. Since both $H_{I, \mathcal{Z}}^{s}\left(\mathbb{R}^{n}\right)$ and $H_{I, \mathcal{Z}}^{s-2}\left(\mathbb{R}^{n}\right)$ are closed subspaces of Banach spaces, they are Banach spaces and the open mapping theorem ensures that also the last part of statement (iii) holds true.

\section{An analyticity result for $(\operatorname{det} q) S_{\mathrm{c}, q, \mathcal{Z}} \circ q$ in Sobolev-Bessel potential spaces}

Theorem 1 Let $\mathbf{c} \in \mathcal{E}$. Let $s \in \mathbb{R}$ be such that $s-2<-n / 2$. Let $\mathcal{Z}$ be a finite subset of $\mathbb{Z}^{n}$. Let $\mathcal{W}$ be an open subset of $\mathbb{D}_{n}^{+}(\mathbb{R})$ such that $\mathbb{Z}(\mathbf{c}, q) \subseteq \mathcal{Z}$ for all $q \in \mathcal{W}$. Then the map $S_{I, \mathbf{c}, \mathcal{Z}}^{\sharp}$ from $\mathcal{W}$ to $H_{I, \mathcal{Z}}^{s}\left(\mathbb{R}^{n}\right)$ defined by

$$
S_{I, \mathbf{c}, \mathcal{Z}}^{\sharp}(q) \equiv(\operatorname{det} q) S_{\mathbf{c}, q, \mathcal{Z}} \circ q \quad \forall q \in \mathcal{W},
$$

is real analytic.

Proof Let $q \in \mathcal{W}$. We first prove that $(\operatorname{det} q) S_{\mathbf{c}, q, \mathcal{Z}} \circ q$ is the only $I$-periodic tempered distribution in $\mathbb{R}^{n}$ which satisfies the following system

$$
\left\{\begin{array}{l}
L_{\mathbf{c}, q}[v]=\sum_{z \in \mathbb{Z}^{n}} \delta_{z}-\sum_{z \in \mathcal{Z}} E_{2 \pi i z} \\
a_{k}(v)=0 \quad \forall k \in \mathcal{Z}
\end{array}\right.
$$

By Lemma 1 (ii), (det $q) S_{\mathbf{c}, q, \mathcal{Z}} \circ q$ satisfies the first equation in (11). Moreover, (2) implies that

$$
a_{k}\left((\operatorname{det} q) S_{\mathbf{c}, q, \mathcal{Z}} \circ q\right)=0 \quad \forall k \in \mathcal{Z} .
$$

Hence, $(\operatorname{det} q) S_{\mathbf{c}, q, \mathcal{Z}} \circ q$ satisfies system (11). Next we assume that $v$ is an $I$-periodic tempered distribution in $\mathbb{R}^{n}$ which satisfies system (11). Then

$$
L_{\mathbf{c}, q}\left[v-(\operatorname{det} q) S_{\mathbf{c}, q, \mathcal{Z}} \circ q\right]=0,
$$

and Proposition 2 (i) ensures that $v-(\operatorname{det} q) S_{\mathbf{c}, q, \mathcal{Z}} \circ q$ belongs to the complex vector space generated by $\left\{E_{2 \pi i k}: k \in \mathbb{Z}(\mathbf{c}, q)\right\}$, and the second equation in (11) ensures that $v-(\operatorname{det} q) S_{\mathbf{c}, q, \mathcal{Z}} \circ q=0$.

Next we show that the right-hand side of the first equation in system (11) belongs to $H_{I}^{s-2}\left(\mathbb{R}^{n}\right)$. Since $s-2<-n / 2$, we have $\delta_{z} \in H^{s-2}\left(\mathbb{R}^{n}\right)$ for all $z \in \mathbb{Z}^{n}$. Hence, $\sum_{z \in \mathbb{Z}^{n}} \delta_{z}$ belongs to $H_{\mathrm{loc}}^{s-2}\left(\mathbb{R}^{n}\right)$. Since $\sum_{z \in \mathbb{Z}^{n}} \delta_{z}$ is obviously $I$-periodic, we also have $\sum_{z \in \mathbb{Z}^{n}} \delta_{z} \in$ $H_{I}^{s-2}\left(\mathbb{R}^{n}\right)$. Accordingly, the right-hand side of the first equation in system (11) belongs to $H_{I}^{s-2}\left(\mathbb{R}^{n}\right)$.

Since $(\operatorname{det} q) S_{\mathbf{c}, q, \mathcal{Z}} \circ q$ is the only tempered distribution which satisfies system (11), the membership of the right-hand side of the first equation in system (11) to $H_{I}^{s-2}\left(\mathbb{R}^{n}\right)$ 
and Proposition 2 imply in particular that $(\operatorname{det} q) S_{\mathbf{c}, q, \mathcal{Z}} \circ q$ belongs to $H_{I}^{s}\left(\mathbb{R}^{n}\right)$ and that $(\operatorname{det} q) S_{\mathbf{c}, q, \mathcal{Z}} \circ q$ is the only element of $H_{I}^{s}\left(\mathbb{R}^{n}\right)$ which satisfies system (11).

Next we consider the map $A$ from $\mathcal{W} \times H_{I, \mathcal{Z}}^{s}\left(\mathbb{R}^{n}\right)$ to the space $H_{I, \mathcal{Z}}^{s-2}\left(\mathbb{R}^{n}\right)$ defined by the equality

$$
A(q, v) \equiv L_{\mathbf{c}, q}[v]-\sum_{z \in \mathbb{Z}^{n}} \delta_{z}+\sum_{z \in \mathcal{Z}} E_{2 \pi i z}
$$

for all $(q, v) \in \mathcal{W} \times H_{I, \mathcal{Z}}^{s}\left(\mathbb{R}^{n}\right)$. By our proof above, the set of zeros of $A$ coincides with the graph of $S_{I, \mathbf{c}, \mathcal{Z}}^{\sharp}$. Moreover, $A$ is real analytic and if $q \in \mathcal{W}$, then partial Fréchet differential $d_{v} A\left(q, S_{I, \mathbf{c}, \mathcal{Z}}^{\sharp}[q]\right)$ of $A$ at the point $\left(q, S_{I, \mathbf{c}, \mathcal{Z}}^{\sharp}[q]\right)$ with respect to the variable $v$ coincides with the map $L_{\mathbf{c}, q}$ from $H_{I, \mathcal{Z}}^{s}\left(\mathbb{R}^{n}\right)$ to $H_{I, \mathcal{Z}}^{s-2}\left(\mathbb{R}^{n}\right)$, which is a linear homeomorphism by Proposition 2 (iii). Then the implicit function theorem in Banach spaces implies that $S_{I, \mathbf{c}, \mathcal{Z}}^{\sharp}$ is real analytic (cf. e.g., Deimling [11, Thm. 15.3]).

\section{Explicit computation of the differentials of a map related to $(\operatorname{det} q) S_{\mathrm{c}, q, \mathcal{Z}} \circ q$ for a particular class of elliptic differential operators}

We now introduce the set

$$
\begin{aligned}
& \mathcal{E}_{0} \equiv\left\{\mathbf{c} \equiv\left(c_{\alpha}\right)_{|\alpha| \leq 2} \in \mathcal{E}: c_{\alpha}=0 \text { if }|\alpha|=1\right. \\
& \left.\quad \text { and } c_{\alpha}=0 \text { if } \alpha=e_{l}+e_{j} \text { with } j, l \in\{1, \ldots, n\}, j \neq l\right\} .
\end{aligned}
$$

Let $\mathbf{c} \in \mathcal{E}_{0}, q \in \mathbb{D}_{n}^{+}(\mathbb{R})$. Since we will soon have to perform computations involving highorder derivatives, we find convenient to set

$$
\tilde{L}_{\mathbf{c}, b}[v] \equiv \sum_{j=1}^{n} b_{j j} c_{j j}^{(2)} \frac{\partial^{2}}{\partial x_{j}^{2}} v+c_{0} v \quad \forall v \in H_{I}^{s}\left(\mathbb{R}^{n}\right),
$$

for all $b \in \mathbb{D}_{n}(\mathbb{R})$ and $s \in \mathbb{R}$. Obviously,

$$
\tilde{L}_{\mathbf{c}, q^{-2}}=L_{\mathbf{c}, q} \quad q \in \mathbb{D}_{n}^{+}(\mathbb{R}), \quad \tilde{L}_{\mathbf{c}, b}=L_{\mathbf{c}, b^{-1 / 2}} \quad \forall b \in \mathbb{D}_{n}^{+}(\mathbb{R}),
$$

where $q^{-2}$ denotes the diagonal matrix with diagonal entries $q_{j j}^{-2}, j=1, \ldots, n$, and where $b^{-1 / 2}$ denotes the diagonal matrix with diagonal entries $b_{j j}^{-1 / 2}, j=1, \ldots, n$. We also note that

$$
L_{\mathbf{c}, q}\left[(\operatorname{det} q) S_{\mathbf{c}, q, \mathcal{Z}} \circ q\right]=L_{\mathbf{c}, b^{-1 / 2}}\left[\left(\operatorname{det} b^{-1 / 2}\right) S_{\mathbf{c}, b^{-1 / 2}, \mathcal{Z}} \circ b^{-1 / 2}\right]
$$

whenever $b=q^{-2}, q \in \mathbb{D}_{n}^{+}(\mathbb{R})$, and that accordingly equality

$$
L_{\mathbf{c}, q}\left[(\operatorname{det} q) S_{\mathbf{c}, q, \mathcal{Z}} \circ q\right]=\sum_{z \in \mathbb{Z}^{n}} \delta_{z}-\sum_{z \in \mathcal{Z}} E_{2 \pi i z} \quad \forall q \in \mathbb{D}_{n}^{+}(\mathbb{R}),
$$

is equivalent to the equality

$$
L_{\mathbf{c}, b^{-1 / 2}}\left[\left(\operatorname{det} b^{-1 / 2}\right) S_{\mathbf{c}, b^{-1 / 2}, \mathcal{Z}} \circ b^{-1 / 2}\right]=\sum_{z \in \mathbb{Z}^{n}} \delta_{z}-\sum_{z \in \mathcal{Z}} E_{2 \pi i z} \quad \forall b \in \mathbb{D}_{n}^{+}(\mathbb{R}),
$$

an equality which we rewrite in the form

$$
\tilde{L}_{\mathbf{c}, b}\left[\left(\operatorname{det} b^{-1 / 2}\right) S_{\mathbf{c}, b^{-1 / 2}, \mathcal{Z}} \circ b^{-1 / 2}\right]=\sum_{z \in \mathbb{Z}^{n}} \delta_{z}-\sum_{z \in \mathcal{Z}} E_{2 \pi i z} \quad \forall b \in \mathbb{D}_{n}^{+}(\mathbb{R}) .
$$


We also note that the following commutativity property holds.

Lemma 2 Let $\mathbf{c}$, $\mathbf{c}^{\sharp} \in \mathcal{E}_{0}$. Let $b \in \mathbb{D}_{n}^{+}(\mathbb{R})$. Let $\mathcal{Z}$ be a finite subset of $\mathbb{Z}^{n}$ such that $\mathbb{Z}\left(\mathbf{c}, b^{-1 / 2}\right) \subseteq \mathcal{Z}$. Let $s \in \mathbb{R}$. Then $\tilde{L}_{\mathbf{c}, b}$ restricts a linear homeomorphism from $H_{I, \mathcal{Z}}^{s}\left(\mathbb{R}^{n}\right)$ onto $H_{I, \mathcal{Z}}^{s-2}\left(\mathbb{R}^{n}\right)$ and

$$
\tilde{L}_{\mathbf{c}, b} \circ\left(\tilde{L}_{\mathbf{c}, b}^{(-1)} \circ \tilde{L}_{\mathbf{c}^{\sharp}, v}\right)[u]=\left(\tilde{L}_{\mathbf{c}, b}^{(-1)} \circ \tilde{L}_{\mathbf{c}^{\sharp}, v}\right) \circ \tilde{L}_{\mathbf{c}, b}[u] \quad \forall u \in H_{I, \mathcal{Z}}^{S}\left(\mathbb{R}^{n}\right),
$$

for all $v \in \mathbb{D}_{n}(\mathbb{R})$.

Proof The first part of the statement is an immediate consequence of Proposition 2 (iii). In order to prove the formula of the statement, we set $u_{1} \equiv \tilde{L}_{\mathbf{c}, b}^{(-1)} \circ \tilde{L}_{\mathbf{c}^{\sharp}, v}[u]$. Then we have $\tilde{L}_{\mathbf{c}, b}\left[u_{1}\right]=\tilde{L}_{\mathbf{c}^{\sharp}, v}[u]$. Since $\tilde{L}_{\mathbf{c}, b} \circ \tilde{L}_{\mathbf{c}^{\sharp}, v}=\tilde{L}_{\mathbf{c}^{\sharp}, v} \circ \tilde{L}_{\mathbf{c}, b}$, we have $\tilde{L}_{\mathbf{c}, b} \circ \tilde{L}_{\mathbf{c}, b}\left[u_{1}\right]=$ $\tilde{L}_{\mathbf{c}^{\sharp}, v} \circ \tilde{L}_{\mathbf{c}, b}[u]$ and thus the formula of the statement follows.

If $\mathbf{c} \in \mathcal{E}_{0}$, we denote by $\mathbf{c}^{*}$ the element of $\mathcal{E}_{0}$ defined by

$$
\mathbf{c}_{\alpha}^{*}=\mathbf{c}_{\alpha} \quad \text { if }|\alpha|=2, \quad \mathbf{c}_{\alpha}^{*}=0 \quad \text { if }|\alpha|<2 .
$$

Then we have the following, which provides a formula for the composite function $S_{I, \mathbf{c}, \mathcal{Z}}^{\times}$of $S_{I, \mathbf{c}, \mathcal{Z}}^{\sharp}$ and of $b^{-1 / 2}$ at the point $b$ computed at $\left(v_{1}, \ldots, v_{j}\right)$ with $v_{1}=\cdots=v_{j} \equiv v$ for all natural numbers $j$, which we need to write the Taylor formula for $S_{I, \mathbf{c}, \mathcal{Z}}^{\times}$at the point $b$.

Proposition 3 Let $\mathbf{c} \in \mathcal{E}_{0}$. Let $s \in \mathbb{R}$ be such that $s-2<-n / 2$. Let $\mathcal{Z}$ be a finite subset of $\mathbb{Z}^{n}$. Let $\widetilde{\mathcal{W}}$ be an open subset of $\mathbb{D}_{n}^{+}(\mathbb{R})$ such that $\mathbb{Z}\left(\mathbf{c}, b^{-1 / 2}\right) \subseteq \mathcal{Z}$ for all $b \in \tilde{\mathcal{W}}$. Let $S_{I, \mathbf{c}, \mathcal{Z}}^{\times}$ be the map from $\widetilde{\mathcal{W}}$ to $H_{I}^{s}\left(\mathbb{R}^{n}\right)$ defined by

$$
S_{I, \mathbf{c}, \mathcal{Z}}^{\times}(b) \equiv S_{I, \mathbf{c}, \mathcal{Z}}^{\sharp}\left(b^{-1 / 2}\right) \quad \forall b \in \tilde{\mathcal{W}} .
$$

Then the following statements hold.

(i) $\tilde{L}_{\mathbf{c}, b}\left[S_{I, \mathbf{c}, \mathcal{Z}}^{\times}(b)\right]=\sum_{z \in \mathbb{Z}^{n}} \delta_{z}-\sum_{z \in \mathcal{Z}} E_{2 \pi i z}$ for all $b \in \tilde{\mathcal{W}}$.

(ii) Let $j \in \mathbb{N} \backslash\{0\}$. The $j$-order differential of the map $S_{I, \mathbf{c}, \mathcal{Z}}^{\times}$at the point $b$ satisfies the equality

$$
\tilde{L}_{\mathbf{c}, b}\left[d^{j} S_{I, \mathbf{c}, \mathcal{Z}}^{\times}(b)[\overbrace{v, \ldots, v}^{j \text { times }}]=-j \tilde{L}_{\mathbf{c}^{*}, v}\left[d^{j-1} S_{I, \mathbf{c}, \mathcal{Z}}^{\times}(b)[\overbrace{v, \ldots, v}^{(j-1) \text { times }}],\right.\right.
$$

for all $b \in \tilde{\mathcal{W}}$ and $v \in \mathbb{D}_{n}(\mathbb{R})$.

(iii) Let $b \in \tilde{\mathcal{W}}$. Then

$$
d^{j} S_{I, \mathbf{c}, \mathcal{Z}}^{\times}(b)[\overbrace{v, \ldots, v}^{j \text { times }}]=(-1)^{j} j !\left(\tilde{L}_{\mathbf{c}, b}^{(-1)} \circ \tilde{L}_{\mathbf{c}^{*}, v}\right)^{j}\left[S_{I, \mathbf{c}, \mathcal{Z}}^{\times}(b)\right] \quad \forall v \in \mathbb{D}_{n}(\mathbb{R}) .
$$

for all $j \in \mathbb{N}$.

(iv) Let $b \in \tilde{\mathcal{W}}$. Then

$\tilde{L}_{\mathbf{c}, b}\left[d^{j} S_{I, \mathbf{c}, \mathcal{Z}}^{\times}(b)[\overbrace{v, \ldots, v}^{j \text { times }}]=(-1)^{j} j !\left(\tilde{L}_{\mathbf{c}, b}^{(-1)} \circ \tilde{L}_{\mathbf{c}^{*}, v}\right)^{j}\left[\sum_{z \in \mathbb{Z}^{n}} \delta_{z}-\sum_{z \in \mathcal{Z}} E_{2 \pi i z}\right]\right.$

for all $v \in \mathbb{D}_{n}(\mathbb{R})$ and $j \in \mathbb{N} \backslash\{0\}$. 
(v) Let $j \in \mathbb{N} \backslash\{0\}$. If $b \in \tilde{\mathcal{W}}$ and $v \in \mathbb{D}_{n}(\mathbb{R})$, then the function $d^{j} S_{I, \mathbf{c}, \mathcal{Z}}^{\times}(b)[v, \ldots, v]$ is real analytic in $\mathbb{R}^{n} \backslash \mathbb{Z}^{n}$.

Proof Statement (i) is an immediate consequence of equality (12). We now prove statement (ii). Since

$$
\tilde{L}_{\mathbf{c}, b}\left[S_{I, \mathbf{c}, \mathcal{Z}}^{\times}(b)\right]=\tilde{L}_{\mathbf{c}^{*}, b}\left[S_{I, \mathbf{c}, \mathcal{Z}}^{\times}(b)\right]+c_{0} S_{I, \mathbf{c}, \mathcal{Z}}^{\times}(b)
$$

and $\tilde{L}_{\mathbf{c}^{*}, b}[v]$ is bilinear in the variable $(b, v)$, and $S_{I, \mathbf{c}, \mathcal{Z}}^{\times}$is differentiable, we can differentiate with respect to $b$ and obtain

$$
\begin{aligned}
d^{j}\left\{\tilde{L}_{\mathbf{c}, b}\left[S_{I, \mathbf{c}, \mathcal{Z}}^{\times}(b)\right]\right\}[v, \ldots, v]= & \left(\begin{array}{c}
j \\
0
\end{array}\right) \tilde{L}_{\mathbf{c}, b}\left[d^{j} S_{I, \mathbf{c}, \mathcal{Z}}^{\times}(b)[v, \ldots, v]\right] \\
& +\left(\begin{array}{c}
j \\
1
\end{array}\right) \tilde{L}_{\mathbf{c}^{*}, v}\left[d^{j-1} S_{I, \mathbf{c}, \mathcal{Z}}^{\times}(b)[v, \ldots, v]\right]
\end{aligned}
$$

for all $(b, v) \in \tilde{\mathcal{W}} \times \mathbb{D}_{n}(\mathbb{R})$ and $j \in \mathbb{N} \backslash\{0\}$. By (i), we have

$$
d^{j}\left\{\tilde{L}_{\mathbf{c}, b}\left[S_{I, \mathbf{c}, \mathcal{Z}}^{\times}(b)\right]\right\}[v, \ldots, v]=0
$$

for all $(b, v) \in \tilde{\mathcal{W}} \times \mathbb{D}_{n}(\mathbb{R})$ and $j \in \mathbb{N} \backslash\{0\}$, and thus statement (ii) holds true.

We now prove statement (iii), and we argue by induction on $j$. If $j=0$, then the statement is obvious. We now assume that the statement holds for $j$, and we prove it for $j+1$. By statement (ii) and by the inductive assumption, we have

$$
\begin{aligned}
\tilde{L}_{\mathbf{c}, b}\left[d^{j+1} S_{I, \mathbf{c}, \mathcal{Z}}^{\times}(b)[v, \ldots, v]\right] & =-(j+1) \tilde{L}_{\mathbf{c}^{*}, v}\left[d^{j} S_{I, \mathbf{c}, \mathcal{Z}}^{\times}(b)[v, \ldots, v]\right] \\
& =-(j+1) \tilde{L}_{\mathbf{c}^{*}, v}\left[(-1)^{j} j !\left(\tilde{L}_{\mathbf{c}, b}^{(-1)} \circ \tilde{L}_{\mathbf{c}^{*}, v}\right)^{j}\left[S_{I, \mathbf{c}, \mathcal{Z}}^{\times}(b)\right]\right] \\
& =(-1)^{j+1}(j+1) ! \tilde{L}_{\mathbf{c}^{*}, v} \circ\left(\tilde{L}_{\mathbf{c}, b}^{(-1)} \circ \tilde{L}_{\mathbf{c}^{*}, v}\right)^{j}\left[S_{I, \mathbf{c}, \mathcal{Z}}^{\times}(b)\right],
\end{aligned}
$$

for all $v \in \mathbb{D}_{n}(\mathbb{R})$. Since $S_{I, \mathbf{c}, \mathcal{Z}}^{\times}$and its differentials have values in $H_{I, \mathcal{Z}}^{s}\left(\mathbb{R}^{n}\right)$, we can apply $\tilde{L}_{\mathbf{c}, b}^{(-1)}$ to both hand sides, and obtain the formula of the statement with $(j+1)$ (cf. Proposition 2 (iii)).

Next we prove statement (iv). By statements (i), (iii) and by Lemma 2, we have

$$
\begin{aligned}
& \tilde{L}_{\mathbf{c}, b}\left[d^{j} S_{I, \mathbf{c}, \mathcal{Z}}^{\times}(b)[v, \ldots, v]\right] \\
& =\tilde{L}_{\mathbf{c}, b}\left[(-1)^{j} j !\left(\tilde{L}_{\mathbf{c}, b}^{(-1)} \circ \tilde{L}_{\mathbf{c}^{*}, v}\right)^{j}\left[S_{I, \mathbf{c}, \mathcal{Z}}^{\times}(b)\right]\right] \\
& =(-1)^{j} j !\left(\tilde{L}_{\mathbf{c}, b}^{(-1)} \circ \tilde{L}_{\mathbf{c}^{*}, v}\right)^{j} \circ \tilde{L}_{\mathbf{c}, b}\left[S_{I, \mathbf{c}, \mathcal{Z}}^{\times}(b)\right] \\
& =(-1)^{j} j !\left(\tilde{L}_{\mathbf{c}, b}^{(-1)} \circ \tilde{L}_{\mathbf{c}^{*}, v}\right)^{j}\left[\sum_{z \in \mathbb{Z}^{n}} \delta_{z}-\sum_{z \in \mathcal{Z}} E_{2 \pi i z}\right],
\end{aligned}
$$

for all $v \in \mathbb{D}_{n}(\mathbb{R})$ and $j \in \mathbb{N} \backslash\{0\}$, and thus statement (iv) holds true. Statement (v) is an immediate consequence of statement (iv), and of the analyticity of $\sum_{z \in \mathbb{Z}^{n}} \delta_{z}-\sum_{z \in \mathcal{Z}} E_{2 \pi i z}$ in $\mathbb{R}^{n} \backslash \mathbb{Z}^{n}$, and of classical elliptic regularity theory. 


\section{Applications to the Helmholtz equation}

\subsection{An analyticity result in Schauder spaces}

In this subsection we consider a concrete application of the results of Sect. 3 to the periodic analog of the fundamental solution of the Helmholtz equation. In order to do so, if $\kappa \in \mathbb{C}$, we define $\mathbf{c}(\kappa)=\left(c_{\alpha}(\kappa)\right)_{|\alpha| \leq 2} \in \mathbb{C}^{N_{2}}$ by setting

$$
c_{e_{j}+e_{j}}(\kappa) \equiv 1, \quad \forall j \in\{1, \ldots, n\},
$$

and

$$
c_{e_{l}+e_{j}}(\kappa) \equiv 0 \quad c_{e_{h}}(\kappa) \equiv 0, \quad \forall h, j, l \in\{1, \ldots, n\}, l \neq j
$$

and

$$
c_{0}(\kappa) \equiv \kappa^{2} .
$$

Thus, if $\kappa \in \mathbb{C}$, we have $\mathbf{c}(\kappa) \in \mathcal{E}_{0}$ and

$$
P[\mathbf{c}(\kappa), D]=\Delta+\kappa^{2} .
$$

Then, a straightforward computation shows that

$$
\mathbb{Z}(\mathbf{c}(\kappa), q) \equiv\left\{z \in \mathbb{Z}^{n}: \kappa^{2}=4 \pi^{2}\left|q^{-1} z\right|^{2}\right\},
$$

and we note that $\mathbb{Z}(\mathbf{c}(\kappa), q)$ is not empty precisely when $-\kappa^{2}$ is an eigenvalue of the Laplace operator in the space of $q$-periodic tempered distributions in $\mathbb{R}^{n}$ (see Proposition 2 (i)). We also note that

$$
\tilde{L}_{\mathbf{c}(\kappa), b}=\sum_{j=1}^{n} b_{j j} \frac{\partial^{2}}{\partial x_{j}^{2}}+\kappa^{2} \quad \forall b \in \mathbb{D}_{n}(\mathbb{R}) .
$$

In order to proceed we need to introduce some Sobolev-Bessel potential spaces on a domain. Let $\Omega$ be an open subset of $\mathbb{R}^{n}$. If $s \in \mathbb{R}$, we denote by $\tilde{H}^{s}(\Omega)$ the closure of $\mathcal{D}(\Omega)$ in $H^{s}\left(\mathbb{R}^{n}\right)$, and we endow $\tilde{H}^{s}(\Omega)$ with the norm of $H^{s}\left(\mathbb{R}^{n}\right)$. Instead, as customary, we denote by $H_{0}^{s}(\Omega)$ the closure of $\mathcal{D}(\Omega)$ in $H^{s}(\Omega)$ (see also McLean [23, p. 77]).

Then we have the following technical statement, which we prove in Appendix.

Theorem 2 Let $\kappa \in \mathbb{C}$. Let $\Omega$ be a bounded open Lipschitz subset of $\mathbb{R}^{n}$. Let $\mathcal{W}$ be an open subset of $\mathbb{D}_{n}^{+}(\mathbb{R})$ such that

$$
\left\{u \in H_{0}^{1}(\Omega): \tilde{L}_{\mathbf{c}(\kappa), b}[u]=0\right\}=\{0\} \quad \forall b \in \mathcal{W} .
$$

Then the following statements hold.

(i) Let $k \in \mathbb{N} \backslash\{0\}$. If $\Omega$ is of class $C^{k-1,1}$ and $b \in \mathcal{W}$, then $\tilde{L}_{\mathbf{c}(\kappa), b}$ is a linear homeomorphism from $H^{k}(\Omega) \cap H_{0}^{1}(\Omega)$ onto $H^{k-2}(\Omega)$.

(ii) Let $k \in \mathbb{N} \backslash\{0\}$. If $\Omega$ is of class $C^{k-1,1}$, then the map $\Phi_{\mathbf{c}(\kappa), k}$ from $\mathcal{W} \times H^{k-2}(\Omega)$ to $H^{k}(\Omega) \cap H_{0}^{1}(\Omega)$ which takes $(b, f)$ to the unique element $v \in H^{k}(\Omega) \cap H_{0}^{1}(\Omega)$ such that $\tilde{L}_{\mathbf{c}(\kappa), b}[v]=f$ is real analytic.

(iii) Let $k \in \mathbb{Z}, k \leq 0$. Let $\Omega$ be of class $C^{1-k, 1}$. If $\left(b^{\sharp}, f^{\sharp}\right) \in \mathcal{W} \times H_{0}^{1}(\Omega)$, then there exists $r \in] 0,+\infty\left[\right.$ such that $\mathrm{cl} \mathbb{B}_{\mathbb{D}_{n}(\mathbb{R})}\left(b^{\sharp}, r\right) \subseteq \mathcal{W}$ and a real analytic map $\tilde{\Phi}_{\mathbf{c}(\kappa), k, b^{\sharp}, f \sharp}$ from $\mathbb{B}_{\mathbb{D}_{n}(\mathbb{R})}\left(b^{\sharp}, r\right) \times \tilde{H}^{k-2}(\Omega)$ to $\tilde{H}^{k}(\Omega)$ such that

$$
\tilde{\Phi}_{\mathbf{c}(\kappa), k, b^{\sharp}, f^{\sharp}}[b, f]=\Phi_{\mathbf{c}(\kappa), 1}[b, f] \quad \forall(b, f) \in \mathbb{B}_{\mathbb{D}_{n}(\mathbb{R})}\left(b^{\sharp}, r\right) \times H_{0}^{1}(\Omega) .
$$


Then we can prove the following.

Theorem 3 Let $m \in \mathbb{N}, \alpha \in] 0,1\left[\right.$. Let $\kappa \in \mathbb{C}$. Let $\mathcal{Z}$ be a finite subset of $\mathbb{Z}^{n}$. Let $\widetilde{\mathcal{W}}$ be an open subset of $\mathbb{D}_{n}^{+}(\mathbb{R})$ such that $\mathbb{Z}\left(\mathbf{c}(\kappa), b^{-1 / 2}\right) \subseteq \mathcal{Z}$ for all $b \in \tilde{\mathcal{W}}$. Let $\Omega$ be a bounded open subset of $\mathbb{R}^{n}$ such that $\operatorname{cl} \Omega \subseteq \mathbb{R}^{n} \backslash \mathbb{Z}^{n}$. Then the map from $\widetilde{\mathcal{W}}$ to $C^{m, \alpha}(\operatorname{cl} \Omega)$, which takes $b$ to the restriction $R_{\Omega} S_{I, \mathbf{c}(\kappa), \mathcal{Z}}^{\times}(b)$ of $S_{I, \mathbf{c}(\kappa), \mathcal{Z}}^{\times}(b)$ to $\Omega$ is real analytic.

Proof Let $s \in \mathbb{Z}$ be such that $s-2<-n / 2$. By the Sobolev imbedding theorem, it suffices to show that the map from $\widetilde{\mathcal{W}}$ to $H^{s+k}(\Omega)$ which takes $b$ to $R_{\Omega} S_{I, \mathbf{c}(\kappa), \mathcal{Z}}^{\times}(b)$ is analytic for all bounded open subsets of $\mathbb{R}^{n}$ such that $\operatorname{cl} \Omega \subseteq \mathbb{R}^{n} \backslash \mathbb{Z}^{n}$ and for all $k \in \mathbb{N}$. Then the validity of the statement of the theorem for any $m \in \mathbb{N}$ and $\alpha \in] 0,1$ [ would follow by a proof which is indeed independent of $m$ and $\alpha$.

Since for every $\Omega$ as above there exists $\zeta \in \mathcal{D}\left(\mathbb{R}^{n} \backslash \mathbb{Z}^{n}\right)$ which equals 1 on an open neighborhood of $\operatorname{cl} \Omega$, it clearly suffices to show that if $k \in \mathbb{N}$, then the map from $\widetilde{\mathcal{W}}$ to $H^{s+k}\left(\mathbb{R}^{n}\right)$ which takes $b$ to $\zeta S_{I, \mathbf{c}(\kappa), \mathcal{Z}}^{\times}(b)$ is analytic for all choices of $\zeta \in \mathcal{D}\left(\mathbb{R}^{n} \backslash \mathbb{Z}^{n}\right)$. Indeed, the restriction operator from $H^{s+k}\left(\mathbb{R}^{n}\right)$ to $H^{s+k}(\Omega)$ is linear and continuous.

Case $k=0$ is an immediate consequence of Theorem 1. Indeed, the multiplication by $\zeta$ is linear and continuous from $H_{I}^{s}\left(\mathbb{R}^{n}\right)$ to $H^{s}\left(\mathbb{R}^{n}\right)$. We now assume that the statement holds for $k$ and we prove it for $k+1$. Let $\zeta \in \mathcal{D}\left(\mathbb{R}^{n} \backslash \mathbb{Z}^{n}\right)$. Let $b_{1} \in \tilde{\mathcal{W}}$. We take an arbitrary bounded open subset $\Omega_{1}$ of $\mathbb{R}^{n}$ of class $C^{\infty}$ such that

$$
\operatorname{supp} \zeta \subseteq \Omega_{1} \subseteq \operatorname{cl} \Omega_{1} \subseteq \mathbb{R}^{n} \backslash \mathbb{Z}^{n}
$$

Possibly replacing $\Omega_{1}$ by a dilation of $\Omega_{1}$ close to the identity, we can assume that $-\kappa^{2}$ is not a Dirichlet eigenvalue for $\sum_{j=1}^{n}\left(b_{1}\right)_{j j} \frac{\partial^{2}}{\partial x_{j}^{2}}$ in $\Omega_{1}$. Indeed the spectrum of the elliptic operator $\sum_{j=1}^{n}\left(b_{1}\right)_{j j} \frac{\partial^{2}}{\partial x_{j}^{2}}$ with Dirichlet boundary conditions in a bounded open subset of $\mathbb{R}^{n}$ of class $C^{\infty}$ is discrete and the eigenvalues scale by a positive factor if the open set undergoes a dilation by a positive factor. Since $-\kappa^{2}$ is not a Dirichlet eigenvalue for $\sum_{j=1}^{n}\left(b_{1}\right)_{j j} \frac{\partial^{2}}{\partial x_{j}^{2}}$ in $\Omega_{1}$, the operator $\tilde{L}_{\mathbf{c}(\kappa), b_{1}}$ is a linear homeomorphism from $H_{0}^{1}\left(\Omega_{1}\right)$ onto $H^{-1}\left(\Omega_{1}\right)$. Since the map from $\widetilde{\mathcal{W}}$ to $\mathcal{L}\left(H_{0}^{1}\left(\Omega_{1}\right), H^{-1}\left(\Omega_{1}\right)\right)$ which takes $b$ to $\tilde{L}_{\mathbf{c}(\kappa), b}$ is linear and continuous and the set of linear homeomorphisms is open, then there exists an open neighborhood $\widetilde{\mathcal{W}}_{1}$ of $b_{1}$ contained in $\tilde{\mathcal{W}}$ such that $\tilde{L}_{\mathbf{c}(\kappa), b}$ is a linear homeomorphism from $H_{0}^{1}\left(\Omega_{1}\right)$ onto $H^{-1}\left(\Omega_{1}\right)$ for all $b \in \widetilde{\mathcal{W}}_{1}$. In particular, condition (19) holds in $\Omega_{1}$ for all $b \in \widetilde{\mathcal{W}}_{1}$.

Since $\Omega_{1}$ is of class $C^{\infty}$ and $\zeta S_{I, \mathbf{c}(\kappa), \mathcal{Z}}^{\times}(b)$ vanishes on an open neighborhood of $\partial \Omega_{1}$ and is of class $C^{\infty}$, we have

$$
\begin{gathered}
\zeta S_{I, \mathbf{c}(\kappa), \mathcal{Z}}^{\times}(b)=\Phi_{\mathbf{c}(\kappa), 1}\left[b, \tilde{L}_{\mathbf{c}(\kappa), b}\left[\zeta S_{I, \mathbf{c}(\kappa), \mathcal{Z}}^{\times}(b)\right]\right], \\
\tilde{L}_{\mathbf{c}(\kappa), b}\left[\zeta S_{I, \mathbf{c}(\kappa), \mathcal{Z}}^{\times}(b)\right] \in \mathcal{D}\left(\Omega_{1}\right) \quad \forall b \in \tilde{\mathcal{W}}_{1} .
\end{gathered}
$$

Next we show that the map from $\tilde{\mathcal{W}}_{1}$ to $H^{s+k-1}\left(\mathbb{R}^{n}\right)$ which takes $b$ to $\tilde{L}_{\mathbf{c}(\kappa), b}\left[\zeta S_{I, \mathbf{c}(\kappa), \mathcal{Z}}^{\times}(b)\right]$ is real analytic. To do so, we note that 


$$
\begin{aligned}
\tilde{L}_{\mathbf{c}(\kappa), b}\left[\zeta(y) S_{I, \mathbf{c}(\kappa), \mathcal{Z}}^{\times}(b)(y)\right]= & \zeta(y) \tilde{L}_{\mathbf{c}(\kappa), b}\left[S_{I, \mathbf{c}(\kappa), \mathcal{Z}}^{\times}(b)(y)\right] \\
& +2 \sum_{j=1}^{n} b_{j j} \frac{\partial \zeta}{\partial x_{j}}(y) \frac{\partial}{\partial x_{j}}\left[S_{I, \mathbf{c}(\kappa), \mathcal{Z}}^{\times}(b)\right](y) \\
& +S_{I, \mathbf{c}(\kappa), \mathcal{Z}}^{\times}(b)(y) \sum_{j=1}^{n} b_{j j} \frac{\partial^{2} \zeta}{\partial x_{j}^{2}}(y) \quad \forall y \in \mathbb{R}^{n},
\end{aligned}
$$

and that

$$
\zeta \tilde{L}_{\mathbf{c}(\kappa), b}\left[S_{I, \mathbf{c}(\kappa), \mathcal{Z}}^{\times}(b)\right]=\left[\sum_{z \in \mathbb{Z}^{n}} \delta_{z}-\sum_{z \in \mathcal{Z}} E_{2 \pi i z}\right] \zeta=-\sum_{z \in \mathcal{Z}} E_{2 \pi i z} \zeta \quad \text { in } \mathbb{R}^{n}
$$

(see Proposition 3 (i)). Thus it suffices to show that each summand in the right-hand side of (23) defines a real analytic map from $\widetilde{\mathcal{W}}_{1}$ to $H^{s+k-1}\left(\mathbb{R}^{n}\right)$.

Since $\zeta \sum_{z \in \mathcal{Z}} E_{2 \pi i z} \in H^{s+k-1}\left(\mathbb{R}^{n}\right)$ is independent of $b$, the first summand in the righthand side of (23) defines a real analytic map from $\widetilde{\mathcal{W}}_{1}$ to $H^{s+k-1}\left(\mathbb{R}^{n}\right)$.

By the membership of $\frac{\partial \zeta}{\partial x_{j}}, \frac{\partial^{2} \zeta}{\partial x_{j}^{2}}$ in $\mathcal{D}\left(\mathbb{R}^{n} \backslash \mathbb{Z}^{n}\right)$ and by inductive assumption, we know that the map from $\tilde{\mathcal{W}}_{1}$ to $H^{s+k-1}\left(\mathbb{R}^{n}\right)$ which takes $b$ to $\frac{\partial \zeta}{\partial x_{j}} \frac{\partial}{\partial x_{j}}\left[S_{I, \mathbf{c}(\kappa), \mathcal{Z}}^{\times}(b)\right]=$ $\frac{\partial}{\partial x_{j}}\left[\frac{\partial \zeta}{\partial x_{j}} S_{I, \mathbf{c}(\kappa), \mathcal{Z}}^{\times}(b)\right]-\frac{\partial^{2} \zeta}{\partial x_{j}^{2}} S_{I, \mathbf{c}(\kappa), \mathcal{Z}}^{\times}(b)$, is real analytic. Hence, the map from $\widetilde{\mathcal{W}}_{1}$ to $H^{s+k-1}\left(\mathbb{R}^{n}\right)$ which takes $b$ to the second summand in the right-hand side of (23) is real analytic.

By the membership of $\frac{\partial^{2} \zeta}{\partial x_{j}^{2}}$ in $\mathcal{D}\left(\mathbb{R}^{n} \backslash \mathbb{Z}^{n}\right)$ and by inductive assumption, we know that the map from $\tilde{\mathcal{W}}_{1}$ to $H^{s+k}\left(\mathbb{R}^{n}\right)$ which takes $b$ to $S_{I, \mathbf{c}(\kappa), \mathcal{Z}}^{\times}(b) \frac{\partial^{2} \zeta}{\partial x_{j}^{2}}$ is analytic. Hence, the map from $\tilde{\mathcal{W}}_{1}$ to $H^{s+k-1}\left(\mathbb{R}^{n}\right)$ which takes $b$ to the third summand in the right-hand side of (23) is real analytic.

Then we conclude that the map from $\widetilde{\mathcal{W}}_{1}$ to $H^{s+k-1}\left(\mathbb{R}^{n}\right)$ which takes $b$ to the right-hand side of (23) is real analytic.

We now discuss separately cases $s+k+1 \geq 1$ and case $s+k+1 \leq 0$. Let $s+k+1 \geq 1$. Since the restriction map is linear and continuous from $H^{s+k-1}\left(\mathbb{R}^{n}\right)$ to $H^{s+k-1}\left(\Omega_{1}\right)$, the map from $\widetilde{\mathcal{W}}_{1}$ to $H^{s+k-1}\left(\Omega_{1}\right)$ which takes $b$ to the right-hand side of (23) is real analytic and Theorem 2 (ii) and equality (22) imply that the map from $\widetilde{\mathcal{W}}_{1}$ to $H^{s+k+1}\left(\Omega_{1}\right)$ which takes $b$ to $\zeta S_{I, \mathbf{c}(\kappa), \mathcal{Z}}^{\times}(b)$ is real analytic. Since $\zeta S_{I, \mathbf{c}(\kappa), \mathcal{Z}}^{\times}(b) \in \mathcal{D}\left(\Omega_{1}\right) \subseteq H_{0}^{s+k+1}\left(\Omega_{1}\right)$ for all $b \in \widetilde{\mathcal{W}}_{1}$ and the operator from $H_{0}^{s+k+1}\left(\Omega_{1}\right)$ to $H^{s+k+1}\left(\mathbb{R}^{n}\right)$ which extends a function to $\mathbb{R}^{n}$ by setting it equal to 0 on $\mathbb{R}^{n} \backslash \Omega$ is linear and continuous, then the map from $\tilde{\mathcal{W}}_{1}$ to $H^{s+k+1}\left(\mathbb{R}^{n}\right)$ which takes $b$ to $\zeta S_{I, \mathbf{c}(\kappa), \mathcal{Z}}^{\times}(b)$ is real analytic (for the extension operator cf. e.g., McLean [23, Thm 3.33].)

Now let $s+k+1 \leq 0$. Since the identity map from the space of the elements of $H^{s+k-1}\left(\mathbb{R}^{n}\right)$ which are of class $C^{\infty}$ and have compact support in $\Omega_{1}$ to $\tilde{H}^{s+k-1}\left(\Omega_{1}\right)$ is isometric, then the map from $\widetilde{\mathcal{W}}_{1}$ to $\tilde{H}^{s+k-1}\left(\Omega_{1}\right)$ which takes $b$ to the right hand side of (23) is real analytic. Here we also note that the right-hand side of (23) belongs to $\mathcal{D}\left(\Omega_{1}\right)$ for all $b \in \widetilde{\mathcal{W}}_{1}$. Then Theorem 2 (iii) and equality (22) imply that the map from $\widetilde{\mathcal{W}}_{1}$ to $\tilde{H}^{s+k+1}\left(\Omega_{1}\right)$ which takes $b$ to $\zeta S_{I, \mathbf{c}(\kappa), \mathcal{Z}}^{\times}(b)$ is real analytic. Since $\tilde{H}^{s+k+1}\left(\Omega_{1}\right)$ is a subspace of $H^{s+k+1}\left(\mathbb{R}^{n}\right)$, the map from $\widetilde{\mathcal{W}}_{1}$ to $H^{s+k+1}\left(\mathbb{R}^{n}\right)$ which takes $b$ to $\zeta S_{I, \mathbf{c}(\kappa), \mathcal{Z}}^{\times}(b)$ is real analytic and the proof is complete. 


\subsection{An analyticity result for the regular part in Roumieu classes}

We now show that the results of the previous sections can be exploited in order to obtain an analyticity result in Roumieu classes.

Theorem 4 Let $\kappa \in \mathbb{C}$. Let $\mathcal{Z}=\{0\}$ if $\kappa=0$ and $\mathcal{Z}=\emptyset$ if $\kappa \neq 0$. Let $\mathcal{Q}$ be a bounded open subset of $\mathbb{D}_{n}^{+}(\mathbb{R})$ such that $\operatorname{cl} \mathcal{Q} \subseteq \mathbb{D}_{n}^{+}(\mathbb{R})$ and that $\mathbb{Z}(\mathbf{c}(\kappa), q) \subseteq \mathcal{Z}$ for all $q \in \operatorname{cl} \mathcal{Q}$. Let $\Omega$ be a bounded open subset of $\mathbb{R}^{n}$ such that

$$
\operatorname{cl} \Omega \subseteq\left(\mathbb{R}^{n} \backslash \mathbb{Z}^{n}\right) \cup\{0\} .
$$

Let $S_{\mathbf{c}(\kappa)}$ be a fundamental solution of $\Delta+\kappa^{2}$. Then there exists $\left.\rho \in\right] 0,+\infty[$ such that the map from $\mathcal{Q}$ to $C_{\omega, \rho}^{0}(\mathrm{cl} \Omega)$ which takes $q$ to $R_{\mathbf{c}(\kappa), q, \mathcal{Z}, S_{\mathbf{c}(\kappa)}}(q \cdot)_{\mid \mathrm{cl} \Omega}$ is real analytic.

Proof Clearly, there is no loss of generality in assuming that $0 \in \Omega$. Let $\Omega^{\prime}$ be a bounded open subset of $\mathbb{R}^{n}$ of class $C^{\infty}$ such that

$$
\Omega \subseteq \operatorname{cl} \Omega \subseteq \Omega^{\prime} \subseteq \operatorname{cl} \Omega^{\prime} \subseteq\left(\mathbb{R}^{n} \backslash \mathbb{Z}^{n}\right) \cup\{0\}
$$

Next we note that $R_{\mathbf{c}(\kappa), q, \mathcal{Z}, S_{\mathbf{c}(\kappa)}} \circ q$, which equals $S_{\mathbf{c}(\kappa), q, \mathcal{Z}} \circ q-S_{\mathbf{c}(\kappa)} \circ q$ in $\mathbb{R}^{n} \backslash \mathbb{Z}^{n}$, is real analytic in $\left(\mathbb{R}^{n} \backslash \mathbb{Z}^{n}\right) \cup\{0\}$ for each fixed value of $q \in \mathbb{D}_{n}^{+}(\mathbb{R})$. We treat separately the case $\kappa=0$ and the case $\kappa \neq 0$. We first consider the case $\kappa=0$. Since $\mathcal{Z}=\{0\}, S_{\mathbf{c}(0), q, \mathcal{Z}} \circ q$ satisfies equation

$$
L_{\mathbf{c}(0), q}\left[S_{\mathbf{c}(0), q, \mathcal{Z} \circ q]}=(\operatorname{det} q)^{-1} \sum_{z \in \mathbb{Z}^{n}} \delta_{z}-\frac{1}{m_{n}(Q)},\right.
$$

[cf. (11)], and we deduce that $R_{\mathbf{c}(0), \mathcal{Z}, S_{\mathbf{c}(0)}}^{\sharp}(q) \equiv R_{\mathbf{c}(0), q, \mathcal{Z}, S_{\mathbf{c}(0)}} \circ q_{\mid \mathrm{cl} \Omega^{\prime}}$ solves the equation

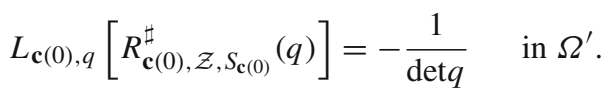

Then by performing a change of variables, we have

$$
\Delta R_{\mathbf{c}(0), q, \mathcal{Z}, S_{\mathbf{c}(0)}}=-\frac{1}{\operatorname{det} q} \quad \text { in } q \Omega^{\prime} .
$$

In order to get rid of the inhomogeneous term in Eq. (24), we set

$$
u_{q}(\xi) \equiv \frac{1}{2 n \operatorname{det} q}|\xi|^{2} \quad \forall \xi \in \mathbb{R}^{n}
$$

and we observe that $\Delta u_{q}=\frac{1}{\operatorname{det} q}$ and we rewrite (24) as

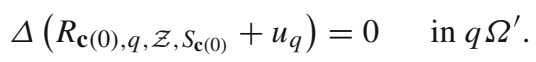

Since $R_{\mathbf{c}(0), q, \mathcal{Z}, S_{\mathbf{c}(0)}}+u_{q}=S_{\mathbf{c}(0), q, \mathcal{Z}}-S_{\mathbf{c}(0)}+u_{q}$ in an open neighborhood of $\partial\left(q \Omega^{\prime}\right)$, then the Green representation formula implies that

$$
\begin{aligned}
& R_{\mathbf{c}(0), q, \mathcal{Z}, S_{\mathbf{c}(0)}}(\xi)+u_{q}(\xi) \\
& =\int_{\partial\left(q \Omega^{\prime}\right)} \frac{\partial}{\partial v_{q \Omega^{\prime}}(\eta)} S_{\mathbf{c}(0)}(\xi-\eta)\left[S_{\mathbf{c}(0), q, \mathcal{Z}}(\eta)-S_{\mathbf{c}(0)}(\eta)+u_{q}(\eta)\right] \mathrm{d} \sigma_{\eta} \\
& -\int_{\partial\left(q \Omega^{\prime}\right)} S_{\mathbf{c}(0)}(\xi-\eta) \frac{\partial}{\partial v_{q \Omega^{\prime}}(\eta)}\left[S_{\mathbf{c}(0), q, \mathcal{Z}}(\eta)-S_{\mathbf{c}(0)}(\eta)+u_{q}(\eta)\right] \mathrm{d} \sigma_{\eta} \quad \forall \xi \in q \Omega^{\prime},
\end{aligned}
$$


and in particular for all $\xi \in q \operatorname{cl} \Omega \subseteq q \Omega^{\prime}$. By elementary calculus, we have

$$
\begin{aligned}
& \mathrm{d} \sigma_{\eta}=\operatorname{det} q\left|q^{-t} v_{\Omega^{\prime}}(y)\right| \mathrm{d} \sigma_{y}, \quad v_{q \Omega^{\prime}}(q y)=\frac{q^{-t} v_{\Omega^{\prime}}(y)}{\left|q^{-t} v_{\Omega^{\prime}}(y)\right|} \\
& \frac{\partial}{\partial v_{q \Omega^{\prime}}}\left[S_{\mathbf{c}(0), q, \mathcal{Z}}-S_{\mathbf{c}(0)}+u_{q}\right](q y) \\
& =\left[D\left(S_{\mathbf{c}(0), q, \mathcal{Z}}-S_{\mathbf{c}(0)}+u_{q}\right)(q y)\right] \cdot v_{q \Omega^{\prime}}(q y) \quad \forall y \in \partial \Omega^{\prime},
\end{aligned}
$$

and thus we obtain

$$
\begin{aligned}
R_{\mathbf{c}(0), q, \mathcal{Z}, S_{\mathbf{c}(0)}}(q x)+u_{q}(q x) \\
=-\int_{\partial \Omega^{\prime}}\left(D S_{\mathbf{c}(0)}(q x-q y)\right) \cdot v_{q \Omega^{\prime}}(q y)\left[S_{\mathbf{c}(0), q, \mathcal{Z}}(q y)-S_{\mathbf{c}(0)}(q y)+u_{q}(q y)\right] \\
\quad \times \operatorname{det} q\left|q^{-t} v_{\Omega^{\prime}}(y)\right| \mathrm{d} \sigma_{y} \\
\quad-\int_{\partial \Omega^{\prime}} S_{\mathbf{c}(0)}(q x-q y)\left[D S_{\mathbf{c}(0), q, \mathcal{Z}}(q y)-D S_{\mathbf{c}(0)}(q y)+D u_{q}(q y)\right] \cdot v_{q \Omega^{\prime}}(q y) \\
\quad \times \operatorname{det} q\left|q^{-t} v_{\Omega^{\prime}}(y)\right| \mathrm{d} \sigma_{y} \\
=-\int_{\partial \Omega^{\prime}}\left(D S_{\mathbf{c}(0)}(q x-q y)\right) \cdot q^{-t} v_{\Omega^{\prime}}(y)\left[S_{\mathbf{c}(0), q, \mathcal{Z}}(q y)-S_{\mathbf{c}(0)}(q y)+u_{q}(q y)\right] \\
\quad \times \operatorname{det} q \mathrm{~d} \sigma_{y} \\
\quad-\int_{\partial \Omega^{\prime}} S_{\mathbf{c}(0)}(q x-q y)\left[D S_{\mathbf{c}(0), q, \mathcal{Z}}(q y)-D S_{\mathbf{c}(0)}(q y)+D u_{q}(q y)\right] \cdot q^{-t} v_{\Omega^{\prime}}(y) \\
\quad \times \operatorname{det} q \mathrm{~d} \sigma_{y},
\end{aligned}
$$

for all $x \in \operatorname{cl} \Omega$. Since $0 \in \Omega \subseteq \Omega^{\prime}$, there exists a bounded open subset $\Omega^{\prime \prime}$ of $\mathbb{R}^{n}$ such that $\partial \Omega^{\prime} \subseteq \Omega^{\prime \prime} \subseteq \operatorname{cl} \Omega^{\prime \prime} \subseteq \mathbb{R}^{n} \backslash \mathbb{Z}^{n}$. By Theorem 3, the map from $\mathbb{D}_{n}^{+}(\mathbb{R})$ to $C^{1}\left(\operatorname{cl} \Omega^{\prime \prime}\right)$ which takes $q$ to $S_{\mathbf{c}(0), q, \mathcal{Z}} \circ q_{\mid \mathrm{cl} \Omega} \Omega^{\prime \prime}$ is real analytic. Since $S_{\mathbf{c}(0)}$ is analytic in $\mathbb{R}^{n} \backslash\{0\}$ and $0 \notin q \mathrm{cl} \Omega^{\prime \prime}$ for all $q \in \mathbb{D}_{n}^{+}(\mathbb{R})$, known results on composition operators imply that the map from $\mathbb{D}_{n}^{+}(\mathbb{R})$ to $C^{1}\left(\operatorname{cl} \Omega^{\prime \prime}\right)$ which takes $q$ to $S_{\mathbf{c}(0)} \circ q_{\mid \mathrm{cl} \Omega^{\prime \prime}}$ is real analytic (cf. Böhme and Tomi [6, p. 10], Henry [14, p. 29], Valent [28, Thm. 5.2, p. 44]). Since $u_{q}(q y)=\frac{1}{2 n \operatorname{det} q}|q y|^{2}$ for all $y \in \operatorname{cl} \Omega^{\prime \prime}$, the map from $\mathbb{D}_{n}^{+}(\mathbb{R})$ to $C^{1}\left(\operatorname{cl} \Omega^{\prime \prime}\right)$ which takes $q$ to $u_{q}(q \cdot)$ is real analytic. Since the restriction operator from $C^{1}\left(\operatorname{cl} \Omega^{\prime \prime}\right)$ to $C^{1}\left(\partial \Omega^{\prime}\right)$ is linear and continuous, the maps

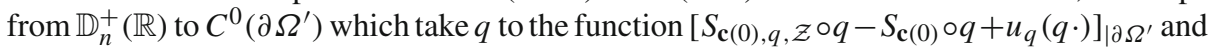
to the function $\left[D S_{\mathbf{c}(0), q, \mathcal{Z}}(q \cdot)-D S_{\mathbf{c}(0)}(q \cdot)+D u_{q}(q \cdot)\right]_{\mid \partial \Omega^{\prime}}$ are real analytic. Then by a result on integral operators with real analytic kernels and with no singularity, for each $\tilde{q} \in \operatorname{cl} \mathcal{Q}$, there exists an open neighborhood $\mathcal{W}_{\tilde{q}}$ of $\tilde{q}$ in $\mathbb{D}_{n}^{+}(\mathbb{R})$ and $\tilde{\rho}>0$ such that the map from $\mathcal{W}_{\tilde{q}}$ to $C_{\omega, \tilde{\rho}}^{0}(\mathrm{cl} \Omega)$ which takes $q$ to the sum of the two integral operators in the right-hand side

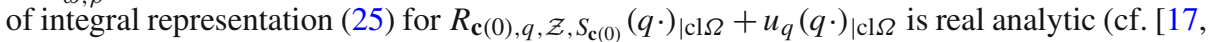
Thm. 3.1]). Since the map from $\mathcal{W}_{\tilde{q}}$ to $C_{\omega, \tilde{\rho}}^{0}(\mathrm{cl} \Omega)$ which takes $q$ to $u_{q}(q \cdot)_{\mid \mathrm{cl} \Omega}$ is obviously real analytic, the map from $\mathcal{W}_{\tilde{q}}$ to $C_{\omega, \tilde{\rho}}^{0}(\operatorname{cl} \Omega)$ which takes $q$ to $R_{\mathbf{c}(0), q, \mathcal{Z}, S_{\mathbf{c}(0)}}(q \cdot)_{\mid \mathrm{cl} \Omega}$ is real analytic. Since the compact set $\mathrm{cl} \mathcal{Q}$ can be covered by a finite family of open neighborhoods as $\mathcal{W}_{\tilde{q}}$, the proof of case $\kappa=0$ is complete.

We now turn to consider case $\kappa \neq 0$. Since $\mathcal{Z}=\emptyset, S_{\mathbf{c}(\kappa), q, \mathcal{Z}} \circ q$ satisfies equation

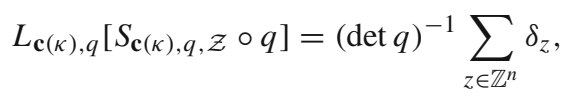


[cf. (11)], and we deduce that $R_{\mathbf{c}(\kappa), \mathcal{Z}, S_{\mathbf{c}(\kappa)}}^{\sharp}(q) \equiv R_{\mathbf{c}(\kappa), q, \mathcal{Z}, S_{\mathbf{c}(\kappa)}} \circ q_{\mid \mathrm{c} 1 \Omega^{\prime}}$ solves the equation

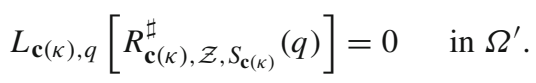

Then by performing a change of variables, we have

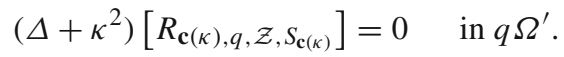

Since $R_{\mathbf{c}(\kappa), q, \mathcal{Z}, S_{\mathbf{c}(\kappa)}}=S_{\mathbf{c}(\kappa), q, \mathcal{Z}}-S_{\mathbf{c}(\kappa)}$ in an open neighborhood of $\partial\left(q \Omega^{\prime}\right)$, then the Green representation formula implies that

$$
\begin{array}{r}
R_{\mathbf{c}(\kappa), q, \mathcal{Z}, S_{\mathbf{c}(\kappa)}}(\xi)=\int_{\partial\left(q \Omega^{\prime}\right)} \frac{\partial}{\partial v_{q} \Omega^{\prime}(\eta)} S_{\mathbf{c}(\kappa)}(\xi-\eta)\left[S_{\mathbf{c}(\kappa), q, \mathcal{Z}}(\eta)-S_{\mathbf{c}(\kappa)}(\eta)\right] \mathrm{d} \sigma_{\eta} \\
-\int_{\partial\left(q \Omega^{\prime}\right)} S_{\mathbf{c}(\kappa)}(\xi-\eta) \frac{\partial}{\partial v_{q} \Omega^{\prime}(\eta)}\left[S_{\mathbf{c}(\kappa), q, \mathcal{Z}}(\eta)-S_{\mathbf{c}(\kappa)}(\eta)\right] \mathrm{d} \sigma_{\eta} \quad \forall \xi \in q \Omega^{\prime},
\end{array}
$$

and in particular for all $\xi \in q \operatorname{cl} \Omega \subseteq q \Omega^{\prime}$. By elementary calculus, we have

$$
\frac{\partial}{\partial v_{q} \Omega^{\prime}}\left[S_{\mathbf{c}(\kappa), q, \mathcal{Z}}-S_{\mathbf{c}(\kappa)}\right](q y)=\left[D\left(S_{\mathbf{c}(\kappa), q, \mathcal{Z}}-S_{\mathbf{c}(\kappa)}\right)(q y)\right] \cdot v_{q \Omega^{\prime}}(q y) \quad \forall y \in \partial \Omega^{\prime},
$$

and thus we obtain

$$
\begin{aligned}
R_{\mathbf{c}(\kappa), q, \mathcal{Z}, S_{\mathbf{c}(\kappa)}}(q x) \\
=-\int_{\partial \Omega^{\prime}}\left(D S_{\mathbf{c}(\kappa)}(q x-q y)\right) \cdot v_{q \Omega^{\prime}}(q y)\left[S_{\mathbf{c}(\kappa), q, \mathcal{Z}}(q y)-S_{\mathbf{c}(\kappa)}(q y)\right] \\
\quad \times \operatorname{det} q\left|q^{-t} v_{\Omega^{\prime}}(y)\right| \mathrm{d} \sigma_{y} \\
\quad-\int_{\partial \Omega^{\prime}} S_{\mathbf{c}(\kappa)}(q x-q y)\left[D S_{\mathbf{c}(\kappa), q, \mathcal{Z}}(q y)-D S_{\mathbf{c}(\kappa)}(q y)\right] \cdot v_{q \Omega^{\prime}}(q y) \\
\quad \times \operatorname{det} q\left|q^{-t} v_{\Omega^{\prime}}(y)\right| \mathrm{d} \sigma_{y} \\
=-\int_{\partial \Omega^{\prime}}\left(D S_{\mathbf{c}(\kappa)}(q x-q y)\right) \cdot q^{-t} v_{\Omega^{\prime}}(y)\left[S_{\mathbf{c}(\kappa), q, \mathcal{Z}}(q y)-S_{\mathbf{c}(\kappa)}(q y)\right] \operatorname{det} q \mathrm{~d} \sigma_{y} \\
\quad-\int_{\partial \Omega^{\prime}} S_{\mathbf{c}(\kappa)}(q x-q y)\left[D S_{\mathbf{c}(\kappa), q, \mathcal{Z}}(q y)-D S_{\mathbf{c}(\kappa)}(q y)\right] \cdot q^{-t} v_{\Omega^{\prime}}(y) \operatorname{det} q \mathrm{~d} \sigma_{y},
\end{aligned}
$$

for all $x \in \operatorname{cl} \Omega$. Since $0 \in \Omega \subseteq \Omega^{\prime}$, there exists a bounded open subset $\Omega^{\prime \prime}$ of $\mathbb{R}^{n}$ such that $\partial \Omega^{\prime} \subseteq \Omega^{\prime \prime} \subseteq \operatorname{cl} \Omega^{\prime \prime} \subseteq \mathbb{R}^{n} \backslash \mathbb{Z}^{n}$. Now let $\tilde{\mathcal{W}}$ be a bounded open neighborhood of $\operatorname{cl} \mathcal{Q}$ in $\mathbb{D}_{n}^{+}(\mathbb{R})$ such that $\mathbb{Z}(\mathbf{c}(\kappa), q) \subseteq \mathcal{Z}$ for all $q \in \operatorname{cl} \tilde{\mathcal{W}}$ and that $\operatorname{cl} \widetilde{\mathcal{W}} \subseteq \mathbb{D}_{n}^{+}(\mathbb{R})$. By Theorem 3 , the map from $\tilde{\mathcal{W}}$ to $C^{1}\left(\mathrm{cl} \Omega^{\prime \prime}\right)$ which takes $q$ to $S_{\mathbf{c}(\kappa), q, \mathcal{Z}} \circ q_{\mid \mathrm{cl}} \Omega^{\prime \prime}$ is real analytic. Since $S_{\mathbf{c}(\kappa)}$ is analytic in $\mathbb{R}^{n} \backslash\{0\}$ and $0 \notin q \mathrm{cl} \Omega^{\prime \prime}$ for all $q \in \mathbb{D}_{n}^{+}(\mathbb{R})$, known results on composition operators imply that the map from $\mathbb{D}_{n}^{+}(\mathbb{R})$ to $C^{1}\left(\mathrm{cl} \Omega^{\prime \prime}\right)$ which takes $q$ to $S_{\mathbf{c}(\kappa)} \circ q_{\mid \mathrm{cl} \Omega} \Omega^{\prime \prime}$ is real analytic (cf. Böhme and Tomi [6, p. 10], Henry [14, p. 29], Valent [28, Thm. 5.2, p. 44]). Since the restriction operator from $C^{1}\left(\operatorname{cl} \Omega^{\prime \prime}\right)$ to $C^{1}\left(\partial \Omega^{\prime}\right)$ is linear and continuous, the maps from $\widetilde{\mathcal{W}}$ to $C^{0}\left(\partial \Omega^{\prime}\right)$ which take $q$ to $\left[S_{\mathbf{c}(\kappa), q, \mathcal{Z}} \circ q-S_{\mathbf{c}(\kappa)} \circ q\right]_{\mid \partial \Omega^{\prime}}$ and to $\left[D S_{\mathbf{c}(\kappa), q, \mathcal{Z}}(q \cdot)-D S_{\mathbf{c}(\kappa)}(q \cdot)\right]_{\mid \partial \Omega^{\prime}}$ are real analytic. Then by a result on integral operators with real analytic kernels and with no singularity, for each $\tilde{q} \in \operatorname{cl} \mathcal{Q}$, there exists an open neighborhood $\mathcal{W}_{\tilde{q}}$ of $\tilde{q}$ contained in $\tilde{\mathcal{W}}$ and $\tilde{\rho}>0$ such that the map from $\mathcal{W}_{\tilde{q}}$ to $C_{\omega, \tilde{\rho}}^{0}(\mathrm{cl} \Omega)$ which takes $q$ to the sum of two integral operators in the right-hand side of integral representation (26) for $R_{\mathbf{c}(\kappa), q, \mathcal{Z}, S_{\mathbf{c}(\kappa)}}(q \cdot)_{\mid \mathrm{cl} \Omega}$ is real analytic (cf. [17, Thm. 3.1]). Since the compact set $\mathrm{cl} \mathcal{Q}$ can be covered by a finite family of open neighborhoods as $\mathcal{W}_{\tilde{q}}$, the proof of case $\kappa \neq 0$ is complete. 
In order to prove the analyticity of $R_{\mathbf{c}(\kappa), q, \mathcal{Z}, S_{\mathbf{c}(\kappa)}}(q x)$ upon $(q, x)$, we need the following technical lemma.

Lemma 3 Let $\Omega$ be a bounded open subset of $\mathbb{R}^{n}$. Let $\mathcal{O}$ be an open subset of $\mathbb{D}_{n}^{+}(\mathbb{R})$. Let $\rho>0$. Let $F$ be a real analytic map from $\mathcal{O}$ to $C_{\omega, \rho}^{0}(\mathrm{cl} \Omega)$. Then the map $\tilde{F}$ from $\mathcal{O} \times \Omega$ to $\mathbb{C}$ defined by

$$
\tilde{F}(q, x) \equiv F(q)(x) \quad \forall(q, x) \in \mathcal{O} \times \Omega,
$$

is real analytic.

Proof We first prove by induction on $m \in \mathbb{N}$ that $\tilde{F}$ is of class $C^{m}(\mathcal{O} \times \Omega)$ and that if $\alpha$, $\beta \in \mathbb{N}^{n},|(\alpha, \beta)|=|\alpha|+|\beta| \leq m$, then

$$
D_{x}^{\beta} D_{q}^{\alpha} F(q)(x)=D_{q}^{\alpha} D_{x}^{\beta} \tilde{F}(q, x) \quad \forall(q, x) \in \mathcal{O} \times \Omega .
$$

We first consider case $m=0$. Since $\alpha=\beta=0$, the statement concerning the derivatives is satisfied by definition of $\tilde{F}$ and we turn to prove the continuity of $\tilde{F}$. Let $(\tilde{q}, \tilde{x}) \in \mathcal{O} \times \Omega$. Let $r \in] 0,+\infty\left[\right.$ be such that $\mathbb{B}_{\mathbb{D}_{n}(\mathbb{R})}(\tilde{q}, r) \times \mathbb{B}_{n}(\tilde{x}, r)$ has closure contained in $\mathcal{O} \times \Omega$. Now let $\epsilon \in] 0,+\infty[$. By the continuity of $F$, there exists $\delta \in] 0, r$ [ such that

$$
|q-\tilde{q}|<\delta \Rightarrow\|F(\tilde{q})-F(q)\|_{C_{\omega, \rho}^{0}(\mathrm{cl} \Omega)}<\epsilon / 2 .
$$

Since $F(\tilde{q})$ is real analytic in $\Omega$, possibly shrinking $\delta$, we can assume that

$$
|x-\tilde{x}|<\delta \Rightarrow|F(\tilde{q})(\tilde{x})-F(\tilde{q})(x)|<\epsilon / 2 .
$$

Then by combining (28) and (29), we conclude that

$$
|x-\tilde{x}|<\delta,|q-\tilde{q}|<\delta \Rightarrow|\tilde{F}(\tilde{q}, \tilde{x})-\tilde{F}(q, x)|=|F(\tilde{q})(\tilde{x})-F(q)(x)|<\epsilon / 2+\epsilon / 2=\epsilon,
$$

and thus $\tilde{F}$ is continuous at $(\tilde{q}, \tilde{x})$.

Next we fix $m \in \mathbb{N}$ and we show that if $\tilde{F}$ is of class $C^{m}$ in $\mathcal{O} \times \Omega$ and if (27) holds true for $m$, then $\tilde{F}$ is of class $C^{m+1}$ in $\mathcal{O} \times \Omega$ and if $\alpha, \beta \in \mathbb{N}^{n},|(\alpha, \beta)|=|\alpha|+|\beta|=m+1$, then (27) is satisfied. It suffices to prove that if $\alpha, \beta \in \mathbb{N}^{n},|(\alpha, \beta)|=|\alpha|+|\beta|=m$, then $D_{q}^{\alpha} D_{x}^{\beta} \tilde{F}$ has first-order continuous partial derivatives with respect to the variables $q_{11}, \ldots$, $q_{n n}, x_{1}, \ldots, x_{n}$ in $\mathcal{O} \times \Omega$ and that

$$
\begin{gathered}
D_{q_{j j}}\left(D_{q}^{\alpha} D_{x}^{\beta} \tilde{F}\right)(q, x)=D_{x}^{\beta} D_{q_{j j}} D_{q}^{\alpha} F(q)(x), \\
D_{x_{j}}\left(D_{q}^{\alpha} D_{x}^{\beta} \tilde{F}\right)(q, x)=D_{x_{j}} D_{x}^{\beta} D_{q}^{\alpha} F(q)(x),
\end{gathered}
$$

for all $(q, x) \in \mathcal{O} \times \Omega$ and $j \in\{1, \ldots, n\}$. Let $(\tilde{q}, \tilde{x}) \in \mathcal{O} \times \Omega$. We first prove that $D_{q_{j j}}\left(D_{q}^{\alpha} D_{x}^{\beta} \tilde{F}\right)$ is continuous at $(\tilde{q}, \tilde{x})$ for all $j \in\{1, \ldots, n\}$. By assumption, $F$ is analytic from $\mathcal{O}$ to $C^{|\beta|+1}(\operatorname{cl} \Omega)$. Since $D_{x}^{\beta}$ is linear and continuous from $C^{|\beta|+1}(\operatorname{cl} \Omega)$ to $C^{1}(\operatorname{cl} \Omega)$, we conclude that $D_{q}^{\alpha} D_{x}^{\beta} F$ is analytic from $\mathcal{O}$ to $C^{1}(\mathrm{cl} \Omega)$. Hence, $D_{q_{j j}}\left(D_{q}^{\alpha} D_{x}^{\beta} F\right)$ exists and is continuous at $\tilde{q}$ as a map from $\mathcal{O}$ to $C^{1}(\operatorname{cl} \Omega)$. Since the limit of the incremental ratio which defines $D_{q_{j j}}\left(D_{q}^{\alpha} D_{x}^{\beta} F\right)$ exists uniformly in $x \in \operatorname{cl} \Omega$ and $D_{q}^{\alpha} D_{x}^{\beta} F=D_{q}^{\alpha} D_{x}^{\beta} \tilde{F}$, we deduce that $D_{q_{j j}}\left(D_{q}^{\alpha} D_{x}^{\beta} \tilde{F}\right)$ exists and equals $D_{q_{j j}}\left(D_{q}^{\alpha} D_{x}^{\beta} F\right)$. Then the same argument we have exploited to prove (30) shows that $D_{q_{j j}} D_{q}^{\alpha} D_{x}^{\beta} \tilde{F}$ is continuous at $(\tilde{q}, \tilde{x})$.

Next we prove that $D_{x_{j}} D_{q}^{\alpha} D_{x}^{\beta} \tilde{F}$ exists and is continuous at $(\tilde{q}, \tilde{x})$. Since $D_{q}^{\alpha} D_{x}^{\beta} F$ is analytic from $\mathcal{O}$ to $C^{1}(\operatorname{cl} \Omega)$ and $D_{x_{j}}$ is linear and continuous from $C^{1}(\operatorname{cl} \Omega)$ to $C^{0}(\operatorname{cl} \Omega)$, 
the map $D_{x_{j}} D_{q}^{\alpha} D_{x}^{\beta} F$ is analytic from $\mathcal{O}$ to $C^{0}(\operatorname{cl} \Omega)$ and $D_{x_{j}} D_{q}^{\alpha} D_{x}^{\beta} F$ exists and is continuous at $\tilde{q}$ as a map from $\mathcal{O}$ to $C^{0}(\operatorname{cl} \Omega)$. Since $D_{q}^{\alpha} D_{x}^{\beta} F=D_{q}^{\alpha} D_{x}^{\beta} \tilde{F}$, we deduce that $D_{x_{j}} D_{q}^{\alpha} D_{x}^{\beta} \tilde{F}=D_{x_{j}} D_{q}^{\alpha} D_{x}^{\beta} F$. Then the same argument we have exploited to prove (30) shows that $D_{x_{j}} D_{q}^{\alpha} D_{x}^{\beta} \tilde{F}=D_{x_{j}} D_{q}^{\alpha} D_{x}^{\beta} F$ is continuous at $(\tilde{q}, \tilde{x})$. Hence, all partial derivatives of $\tilde{F}$ up to order $m+1$ are continuous, and $\tilde{F}$ is of class $C^{m+1}$.

We now prove the first equality of (31). If $\beta=0$, the first equality of (31) is obvious. We now assume that $\beta \neq 0$. There is no loss of generality in assuming that $\beta_{1}>0$. Since $\tilde{F}$ is of class $C^{m+1}$, inequality (27) in case $|(\alpha, \beta)| \leq m$ implies that

$$
\begin{aligned}
& D_{q_{j j}}\left(D_{q}^{\alpha} D_{x}^{\beta} \tilde{F}\right)(q, x)=D_{x}^{e_{1}} D_{x}^{\beta-e_{1}} D_{q_{j j}} D_{q}^{\alpha} \tilde{F}(q, x) \\
& \quad=D_{x}^{e_{1}} D_{x}^{\beta-e_{1}} D_{q_{j j}} D_{q}^{\alpha} F(q)(x)=D_{x}^{\beta} D_{q_{j j}} D_{q}^{\alpha} F(q)(x) \quad \forall(q, x) \in \mathcal{O} \times \Omega,
\end{aligned}
$$

and thus the first equality of (31) holds true.

We now prove the second equality of (31). By the analyticity of $D_{q}^{\alpha} F$ from $\mathcal{O}$ to $C^{|\beta|+1}(\operatorname{cl} \Omega)$ and by the linearity and continuity of $D_{x}^{\beta}$ from $C^{|\beta|+1}(\operatorname{cl} \Omega)$ to $C^{1}(\operatorname{cl} \Omega)$, the map $D_{x}^{\beta} D_{q}^{\alpha} F$ is analytic from $\mathcal{O}$ to $C^{1}(\mathrm{cl} \Omega)$. Moreover the inductive assumption implies that (27) holds. Since $D_{x}^{\beta} D_{q}^{\alpha} F(q) \in C^{1}(\operatorname{cl} \Omega)$ and $\tilde{F}$ is of class $C^{m+1}$, we can take the $D_{x_{j}}$ derivative in both hand sides of (27) and obtain the second equality of (31). Thus the proof of our inductive argument is complete. Then $\tilde{F} \in C^{\infty}(\mathcal{O} \times \Omega)$ and we now turn to prove that $\tilde{F}$ is analytic. By a known analyticity criterion, it suffices to show that if $K_{1}$ and $K_{2}$ are compact subsets of $\mathcal{O}$ and $\Omega$, respectively, then there exists $r \in] 0,+\infty[$ such that

$$
\sup _{\alpha, \beta \in \mathbb{N}^{n}} \frac{r^{|\alpha+\beta|}}{|\alpha+\beta| !} \sup _{(q, x) \in K_{1} \times K_{2}}\left|D_{q}^{\alpha} D_{x}^{\beta} \tilde{F}(q, x)\right|<+\infty .
$$

Since $F$ is analytic, then there exist $\left.r_{1}, M_{1} \in\right] 0,+\infty[$ such that

$$
\sup _{\alpha \in \mathbb{N}^{n}} \frac{r_{1}^{|\alpha|}}{|\alpha| !}\left\|D_{q}^{\alpha} F(q)\right\|_{C_{\omega, \rho}^{0}(\mathrm{cl} \Omega)} \leq M_{1}
$$

for all $q \in K_{1}$. Hence,

$$
\frac{r_{1}^{|\alpha|}}{|\alpha| !} \frac{\rho^{|\beta|}}{|\beta| !} \sup _{x \in \mathrm{c} 1 \Omega}\left|D_{x}^{\beta} D_{q}^{\alpha} \tilde{F}(q, x)\right| \leq M_{1}
$$

for all $\alpha, \beta \in \mathbb{N}^{n}, q \in K_{1}$. Since $|\alpha| !|\beta| ! \leq|\alpha+\beta|$ !, we have

$$
\frac{\left(\min \left\{r_{1}, \rho\right\}\right)^{|\alpha+\beta|}}{|\alpha+\beta| !}\left|D_{x}^{\beta} D_{q}^{\alpha} \tilde{F}(q, x)\right| \leq M_{1}
$$

for all $(q, x) \in K_{1} \times K_{2}$ and for all $\alpha, \beta \in \mathbb{N}^{n}$. Hence, $\tilde{F}$ is real analytic.

Theorem 4 and Lemma 3 imply the validity of the following.

Theorem 5 Let $\kappa \in \mathbb{C}$. Let $\mathcal{Z}=\{0\}$ if $\kappa=0$ and $\mathcal{Z}=\emptyset$ if $\kappa \neq 0$. Let $\mathcal{Q}$ be an open subset of $\mathbb{D}_{n}^{+}(\mathbb{R})$ such that $\mathbb{Z}(\mathbf{c}(\kappa), q) \subseteq \mathcal{Z}$ for all $q \in \mathcal{Q}$. Let $S_{\mathbf{c}(\kappa)}$ be a fundamental solution of $\Delta+\kappa^{2}$.

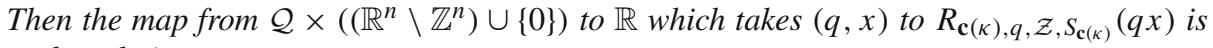
real analytic.

Then we have the following. 
Theorem 6 Let $\kappa \in \mathbb{C}$. Let $\mathcal{Z}=\{0\}$ if $\kappa=0$ and $\mathcal{Z}=\emptyset$ if $\kappa \neq 0$. Let $q_{0} \in \mathbb{D}_{n}^{+}(\mathbb{R})$ be such that $\mathbb{Z}\left(\mathbf{c}(\kappa), q_{0}\right) \subseteq \mathcal{Z}$. Let $\Omega^{\prime}$ be a bounded open subset of $\mathbb{R}^{n}$ such that

$$
\operatorname{cl} \Omega^{\prime} \subseteq\left(\mathbb{R}^{n} \backslash q_{0} \mathbb{Z}^{n}\right) \cup\{0\} .
$$

Let $S_{\mathbf{c}(\kappa)}$ be a fundamental solution of $\Delta+\kappa^{2}$. Then there exist $\left.\rho^{\prime} \in\right] 0,+\infty[$ and an open neighborhood $\mathcal{Q}_{0}$ of $q_{0}$ in $\mathbb{D}_{n}^{+}(\mathbb{R})$ such that

$$
\mathbb{Z}(\mathbf{c}(\kappa), q) \subseteq \mathcal{Z} \quad \forall q \in \operatorname{cl} \mathcal{Q}_{0}, \quad \operatorname{cl} \Omega^{\prime} \subseteq\left(\mathbb{R}^{n} \backslash q \mathbb{Z}^{n}\right) \cup\{0\} \quad \forall q \in \mathcal{Q}_{0},
$$

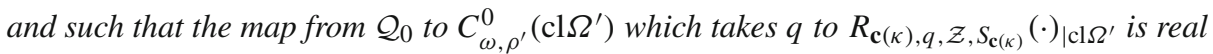
analytic.

Proof By assumption $q_{0}^{-1} \mathrm{cl} \Omega^{\prime} \subseteq\left(\mathbb{R}^{n} \backslash \mathbb{Z}^{n}\right) \cup\{0\}$. Let $\Psi$ be the map from $\mathbb{D}_{n}^{+}(\mathbb{R}) \times \mathbb{R}^{n}$ to $\mathbb{R}^{n}$ defined by

$$
\Psi(q, x) \equiv q^{-1} x \quad \forall(q, x) \in \mathbb{D}_{n}^{+}(\mathbb{R}) \times \mathbb{R}^{n} .
$$

Since $\Psi$ is continuous, the set $\Psi^{\leftarrow}\left(\left(\mathbb{R}^{n} \backslash \mathbb{Z}^{n}\right) \cup\{0\}\right)$ is an open neighborhood of the compact set $\left\{q_{0}\right\} \times \operatorname{cl} \Omega^{\prime}$. Next we endow $\mathbb{D}_{n}^{+}(\mathbb{R}) \times \mathbb{R}^{n}$ by the distance defined by

$$
d\left(\left(q_{1}, x_{1}\right),\left(q_{2}, x_{2}\right)\right)=\left|q_{1}-q_{2}\right|+\left|x_{1}-x_{2}\right| \quad \forall\left(q_{1}, x_{1}\right),\left(q_{2}, x_{2}\right) \in \mathbb{D}_{n}^{+}(\mathbb{R}) \times \mathbb{R}^{n} .
$$

Let $\delta \in] 0,+\infty$ [ be less than the distance between $\left\{q_{0}\right\} \times \operatorname{cl} \Omega^{\prime}$ and the complement of $\Psi^{\leftarrow}\left(\left(\mathbb{R}^{n} \backslash \mathbb{Z}^{n}\right) \cup\{0\}\right)$. Then we have $\operatorname{cl} \mathbb{B}_{\mathbb{D}_{n}(\mathbb{R})}\left(q_{0}, \delta\right) \times \operatorname{cl} \Omega^{\prime} \subseteq \Psi^{\leftarrow}\left(\left(\mathbb{R}^{n} \backslash \mathbb{Z}^{n}\right) \cup\{0\}\right)$, and accordingly the second inclusion in (32) holds for all $q \in \operatorname{cl} \mathbb{B}_{\mathbb{D}_{n}(\mathbb{R})}\left(q_{0}, \delta\right)$. If $\kappa=0$, then $\mathbb{Z}(\mathbf{c}(\kappa), q)=\{0\}=\mathcal{Z}$ for all $q \in \mathbb{D}_{n}^{+}(\mathbb{R})$, and in particular for all $q \in \operatorname{cl} \mathbb{B}_{\mathbb{D}_{n}(\mathbb{R})}\left(q_{0}, \delta\right)$. We now show that if $\kappa \neq 0$, then possibly taking a smaller $\delta$, we can assume that $\mathbb{Z}(\mathbf{c}(\kappa), q)=$ $\emptyset=\mathcal{Z}$ for all $q \in \operatorname{cl} \mathbb{B}_{\mathbb{D}_{n}(\mathbb{R})}\left(q_{0}, \delta\right)$. Let $\left.\delta_{1} \in\right] 0,+\infty\left[\right.$ be such that $\operatorname{cl} \mathbb{B}_{\mathbb{D}_{n}(\mathbb{R})}\left(q_{0}, \delta_{1}\right) \subseteq \mathbb{D}_{n}^{+}(\mathbb{R})$. By (18), we have

$$
\begin{aligned}
\mathbb{Z}(\mathbf{c}(\kappa), q) & \subseteq \mathbb{Z}^{n} \cap \operatorname{cl} \mathbb{B}_{n}\left(0, \rho_{1}\right) \\
\text { with } \quad \rho_{1} & \equiv|\kappa|\left(2 \pi \min _{q \in \mathrm{cl} \mathbb{B}_{\mathbb{D}_{n}(\mathbb{R})}\left(q_{0}, \delta_{1}\right)} \min _{j \in\{1, \ldots, n\}} q_{j j}^{-1}\right)^{-1}
\end{aligned}
$$

for all $q \in \operatorname{cl} \mathbb{B}_{\mathbb{D}_{n}(\mathbb{R})}\left(q_{0}, \delta_{1}\right)$. Since $\mathbb{Z}\left(\mathbf{c}(\kappa), q_{0}\right)=\varnothing$, the continuous function $\kappa^{2}-$ $4 \pi^{2}\left|q_{0}^{-1} z\right|^{2}$ does not vanish for $z$ in the compact set $\mathbb{Z}^{n} \cap \mathrm{cl} \mathbb{B}_{n}\left(0, \rho_{1}\right)$. Hence, possibly taking a smaller $\delta$, we may assume that $\kappa^{2}-4 \pi^{2}\left|q^{-1} z\right|^{2}$ does not vanish for $z \in \mathbb{Z}^{n} \cap \operatorname{cl}_{n}\left(0, \rho_{1}\right)$ when $q \in \operatorname{clB}_{\mathbb{B}_{n}(\mathbb{R})}\left(q_{0}, \delta\right)$. Hence, the inclusion in (33) implies that $\mathbb{Z}(\mathbf{c}(\kappa), q)=\varnothing$ for all $q \in \operatorname{cl} \mathbb{B}_{\mathbb{D}_{n}(\mathbb{R})}\left(q_{0}, \delta\right)$. By Theorem 5 and by equality $R_{\mathbf{c}(\kappa), q, \mathcal{Z}, S_{\mathbf{c}(\kappa)}}(x)=R_{\mathbf{c}(\kappa), q, \mathcal{Z}, S_{\mathbf{c}(\kappa)}}(q \Psi(q, x))$, the map which takes the pair $(q, x)$ to $R_{\mathbf{c}(\kappa), q, \mathcal{Z}, S_{\mathbf{c}(\kappa)}}(x)$ is analytic in an open neighborhood of $\operatorname{cl}\left(\mathbb{B}_{\mathbb{D}_{n}(\mathbb{R})}\left(q_{0}, \delta\right) \times \Omega^{\prime}\right)$. Then there

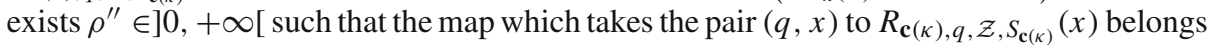
to $C_{\omega, \rho^{\prime \prime}}^{0}\left(\mathrm{cl}\left(\mathbb{B}_{\mathbb{D}_{n}(\mathbb{R})}\left(q_{0}, \delta\right) \times \Omega^{\prime}\right)\right)$. Then one can prove that the map from $\mathbb{B}_{\mathbb{D}_{n}(\mathbb{R})}\left(q_{0}, \delta\right)$ to $C_{\omega, \rho^{\prime}}^{0}\left(\mathrm{cl} \Omega^{\prime}\right)$ which takes $q$ to $R_{\mathbf{c}(\kappa), q, \mathcal{Z}, S_{\mathbf{c}(\kappa)}}(\cdot)_{\mid \mathrm{cl} \Omega^{\prime}}$ is real analytic for all $\left.\rho^{\prime} \in\right] 0, \rho^{\prime \prime}[(\mathrm{cf}$. [8, Prop. A1]).

\subsection{Two more analyticity results for $S_{\mathrm{c}(\kappa), q, \mathcal{Z}} \circ q$}

Since $S_{\mathbf{c}(\kappa), q, \mathcal{Z}}=S_{\mathbf{c}(\kappa)}+R_{\mathbf{c}(\kappa), q, \mathcal{Z}, S_{\mathbf{c}(\kappa)}}$, and $S_{\mathbf{c}(\kappa)}$ is analytic in $\mathbb{R}^{n} \backslash\{0\}$, Theorem 5 implies the validity of the following. 
Theorem 7 Let $\kappa \in \mathbb{C}$. Let $\mathcal{Z}=\{0\}$ if $\kappa=0$ and $\mathcal{Z}=\emptyset$ if $\kappa \neq 0$. Let $\mathcal{Q}$ be an open subset of $\mathbb{D}_{n}^{+}(\mathbb{R})$ such that $\mathbb{Z}(\mathbf{c}(\kappa), q) \subseteq \mathcal{Z}$ for all $q \in \mathcal{Q}$. Then the map from $\mathcal{Q} \times\left(\mathbb{R}^{n} \backslash \mathbb{Z}^{n}\right)$ to $\mathbb{R}$ which takes $(q, x)$ to $S_{\mathbf{c}(\kappa), q, \mathcal{Z}}(q x)$ is real analytic.

Example 1 If we choose $\kappa=0$ in (15)-(17), then we have $P[\mathbf{c}(0), D]=\Delta$ and $\mathbb{Z}(\mathbf{c}(0), q)=$ $\{0\}$ for all $q \in \mathbb{D}_{n}^{+}(\mathbb{R})$. Then $S_{\mathbf{c}(0), q, \mathcal{Z}}(x)$ with $\mathcal{Z}=\{0\}$ equals the sum of the series in (4) and Theorem 7 ensures the analyticity of $S_{\mathbf{c}(0), q, \mathcal{Z}}(q x)$ in $(q, x) \in \mathbb{D}_{n}^{+}(\mathbb{R}) \times\left(\mathbb{R}^{n} \backslash \mathbb{Z}^{n}\right)$, and accordingly the analyticity of the sum of the series in (4) in the set of $(q, x) \in \mathbb{D}_{n}^{+}(\mathbb{R}) \times \mathbb{R}^{n}$ such that $q^{-1} x \notin \mathbb{Z}^{n}$, i.e., jointly in the variables $q$ and $x$.

Example 2 If we choose $\kappa \neq 0$ in (15)-(17) and if we take an open subset $\mathcal{Q}$ of $\mathbb{D}_{n}^{+}(\mathbb{R})$ such that $-\kappa^{2}$ is not an eigenvalue of $\Delta$ in the space of $q$-periodic distributions in $\mathbb{R}^{n}$ for all $q \in \mathcal{Q}$, we have $P[\mathbf{c}(\kappa), D]=\Delta+\kappa^{2}$ and $\mathbb{Z}(\mathbf{c}(\kappa), q)=\emptyset$ for all $q \in \mathcal{Q}$. Then $S_{\mathbf{c}(\kappa), q, \mathcal{Z}}(x)$ with $\mathcal{Z}=\emptyset$ equals the sum of the following series

$$
-\sum_{z \in \mathbb{Z}^{n}} \frac{1}{m_{n}(Q)\left(4 \pi^{2}\left|q^{-1} z\right|^{2}-\kappa^{2}\right)} e^{2 \pi i\left(q^{-1} z\right) \cdot x},
$$

and Theorem 7 ensures the analyticity of $S_{\mathbf{c}(\kappa), q, \mathcal{Z}}(q x)$ in $(q, x) \in \mathcal{Q} \times\left(\mathbb{R}^{n} \backslash \mathbb{Z}^{n}\right)$, and accordingly the analyticity of the sum of the series in (34) in the set of $(q, x) \in \mathcal{Q} \times \mathbb{R}^{n}$ such that $q^{-1} x \notin \mathbb{Z}^{n}$, i.e., jointly in the variables $q$ and $x$.

Finally, we have the following.

Theorem 8 Let $\kappa \in \mathbb{C}$. Let $\mathcal{Z}=\{0\}$ if $\kappa=0$ and $\mathcal{Z}=\emptyset$ if $\kappa \neq 0$. Let $\mathcal{Q}$ be a bounded open subset of $\mathbb{D}_{n}^{+}(\mathbb{R})$ such that $\operatorname{cl} \mathcal{Q} \subseteq \mathbb{D}_{n}^{+}(\mathbb{R})$ and that $\mathbb{Z}(\mathbf{c}(\kappa), q) \subseteq \mathcal{Z}$ for all $q \in \operatorname{cl} \mathcal{Q}$. Let $\Omega$ be a bounded open subset of $\mathbb{R}^{n}$ such that

$$
\operatorname{cl} \Omega \subseteq \mathbb{R}^{n} \backslash \mathbb{Z}^{n}
$$

Then there exists $\rho \in] 0,+\infty\left[\right.$ such that the map from $\mathcal{Q}$ to $C_{\omega, \rho}^{0}(\mathrm{cl} \Omega)$ which takes $q$ to the function $S_{\mathbf{c}(\kappa), q, \mathcal{Z}}(q \cdot)_{\mid \mathrm{c} l \Omega}$ is real analytic.

Proof Let $S_{\mathbf{c}(\kappa)}$ be a fundamental solution of $\Delta+\kappa^{2}$. Since $S_{\mathbf{c}(\kappa)}(q x)$ is analytic in an open neighborhood of the $\operatorname{set} \operatorname{cl}(\mathcal{Q} \times \Omega)$, then there exists $\left.\rho^{\prime} \in\right] 0,+\infty\left[\operatorname{such}\right.$ that $S_{\mathbf{c}(\kappa)}(q x) \in$ $C_{\omega, \rho^{\prime}}^{0}(\operatorname{cl}(\mathcal{Q} \times \Omega))$. Then the map from $\mathcal{Q}$ to $C_{\omega, \rho}^{0}(\operatorname{cl} \Omega)$, which takes $q$ to $S_{\mathbf{c}(\kappa)}(q \cdot)_{\mid \mathrm{cl} \Omega}$ is real analytic for all $\rho \in] 0, \rho^{\prime}\left[\right.$ (cf. [8, Prop. A1]). Then equality $S_{\mathbf{c}(\kappa), q, \mathcal{Z}}=S_{\mathbf{c}(\kappa)}+R_{\mathbf{c}(\kappa), q, \mathcal{Z}, S_{\mathbf{c}(\kappa)}}$ and Theorem 4 imply the validity of the statement.

\section{Appendix}

We now prove technical Theorem 2, and we first consider statements (i) and (ii).

Proof (of statements ( $i$ ) and (ii) of Theorem 2) Statement (i) follows by condition (19) and by classical elliptic regularity theory (cf. e.g., Lions and Magenes [21, Thm. 5.4, p. 165]). We now prove statement (ii). We consider the map $A$ from $\mathcal{W} \times H^{k-2}(\Omega) \times\left(H^{k}(\Omega) \cap H_{0}^{1}(\Omega)\right)$ to the space $H^{k-2}(\Omega)$ defined by the equality

$$
A(b, f, v) \equiv \tilde{L}_{\mathbf{c}(\kappa), b}[v]-f
$$

for all $(b, f, v) \in \mathcal{W} \times H^{k-2}(\Omega) \times\left(H^{k}(\Omega) \cap H_{0}^{1}(\Omega)\right)$. By definition, the set of zeros of $A$ coincides with the graph of $\Phi_{\mathbf{c}(\kappa), k}$. Moreover, $A$ is real analytic and if $(b, f) \in$ 
$\mathcal{W} \times H^{k-2}(\Omega)$, then the partial Fréchet differential $d_{v} A\left(b, f, \Phi_{\mathbf{c}(\kappa), k}[b, f]\right)$ of $A$ at the point $\left(b, f, \Phi_{\mathbf{c}(\kappa), k}[b, f]\right)$ with respect to the variable $v$ coincides with the map $\tilde{L}_{\mathbf{c}(\kappa), b}$ from $H^{k}(\Omega) \cap H_{0}^{1}(\Omega)$ to $H^{k-2}(\Omega)$, which is a linear homeomorphism by statement (i). Then the implicit function theorem in Banach spaces implies that $\Phi_{\mathbf{c}(\kappa), k}$ is real analytic (cf. e.g., Deimling [11, Thm. 15.3]).

Next we plan to compute the Taylor expansion of $\Phi_{\mathbf{c}(\kappa), 1}$ around each point of its domain. Since $\Phi_{\mathbf{c}(\kappa), 1}$ is linear in the second variable $f$, it suffices to compute the partial differential of order $j$ of $\Phi_{\mathbf{c}(\kappa), 1}$ with respect to $b$ at the point $\left(v_{1}, \ldots, v_{j}\right)$ with $v_{1}=\cdots=v_{j} \equiv v$ for all natural numbers $j$. We do so by means of the following.

Proposition 4 Let $\kappa \in \mathbb{C}$. Let $\Omega$ be a bounded open Lipschitz subset of $\mathbb{R}^{n}$. Let $\mathcal{W}$ be an open subset of $\mathbb{D}_{n}^{+}(\mathbb{R})$ such that condition (19) holds true. Let $f \in H^{-1}(\Omega)$. Let $\phi_{\mathbf{c}(\kappa), f}^{\times}$be the map from $\mathcal{W}$ to $H_{0}^{1}(\Omega)$ defined by

$$
\phi_{\mathbf{c}(\kappa), f}^{\times}(b) \equiv \Phi_{\mathbf{c}(\kappa), 1}[b, f] \quad \forall b \in \mathcal{W} .
$$

Then the following statements hold.

(i) $\tilde{L}_{\mathbf{c}(\kappa), b}\left[\phi_{\mathbf{c}(\kappa), f}^{\times}(b)\right]=f$ for all $b \in \mathcal{W}$.

(ii) Let $j \in \mathbb{N} \backslash\{0\}$. The $j$-order differential of the map $\phi_{\mathbf{c}(\kappa), f}^{\times}(b)$ at the point $b$ satisfies the equality

$$
\tilde{L}_{\mathbf{c}(\kappa), b}\left[d^{j} \phi_{\mathbf{c}(\kappa), f}^{\times}(b)[\overbrace{v, \ldots, v}^{j \text { times }}]\right]=-j \tilde{L}_{\mathbf{c}(\kappa)^{*}, v}\left[d^{j-1} \phi_{\mathbf{c}(\kappa), f}^{\times}(b)[\overbrace{v, \ldots, v}^{(j-1) \text { times }}],\right.
$$

for all $b \in \mathcal{W}$ and $v \in \mathbb{D}_{n}(\mathbb{R})$.

(iii) Let $b \in \mathcal{W}$. Then

$d^{j} \phi_{\mathbf{c}(\kappa), f}^{\times}(b)[\overbrace{v, \ldots, v}^{j \text { times }}]=(-1)^{j} j !\left(\tilde{L}_{\mathbf{c}(\kappa), b}^{(-1)} \circ \tilde{L}_{\mathbf{c}(\kappa)^{*}, v}\right)^{j}\left[\phi_{\mathbf{c}(\kappa), f}^{\times}(b)\right] \quad \forall v \in \mathbb{D}_{n}(\mathbb{R})$.

for all $j \in \mathbb{N}$.

Proof The proof follows the lines of that of Proposition 3. Statement (i) is an immediate consequence of the definition of $\phi_{\mathbf{c}(\kappa), f}^{\times}$. We now prove statement (ii). Since

$$
\tilde{L}_{\mathbf{c}(\kappa), b}\left[\phi_{\mathbf{c}(\kappa), f}^{\times}(b)\right]=\tilde{L}_{\mathbf{c}(\kappa)^{*}, b}\left[\phi_{\mathbf{c}(\kappa), f}^{\times}(b)\right]+\kappa^{2} \phi_{\mathbf{c}(\kappa), f}^{\times}(b)
$$

and $\tilde{L}_{\mathbf{c}(\kappa)^{*}, b}[v]$ is bilinear in the variable $(b, v)$, and $\phi_{\mathbf{c}(\kappa), f}^{\times}$is differentiable in $b$, standard differentiation rules imply the validity of formula (13) with $S_{I, \mathbf{c}, \mathcal{Z}}^{\times}(b), \mathbf{c}$ and $\mathbf{c}^{*}$ replaced by $\phi_{\mathbf{c}(\kappa), f}^{\times}(b), \mathbf{c}(\kappa)$ and $\mathbf{c}(\kappa)^{*}$, respectively, for all $(b, v) \in \tilde{\mathcal{W}} \times \mathbb{D}_{n}(\mathbb{R})$ and $j \in \mathbb{N} \backslash\{0\}$. By (i), we have

$$
d^{j}\left\{\tilde{L}_{\mathbf{c}(\kappa), b}\left[\phi_{\mathbf{c}(\kappa), f}^{\times}(b)\right]\right\}[v, \ldots, v]=0
$$

for all $(b, v) \in \widetilde{\mathcal{W}} \times \mathbb{D}_{n}(\mathbb{R})$ and $j \in \mathbb{N} \backslash\{0\}$, and thus statement (ii) holds true.

We now prove statement (iii), and we argue by induction on $j$. If $j=0$, then the statement is obvious. We now assume that the statement holds for $j$ and we prove it for $j+1$. By statement (ii) and by the inductive assumption, we deduce the validity of formula (14) with $S_{I, \mathbf{c}, \mathcal{Z}}^{\times}(b), \mathbf{c}$ and $\mathbf{c}^{*}$ replaced by $\phi_{\mathbf{c}(\kappa), f}^{\times}(b), \mathbf{c}(\kappa)$ and $\mathbf{c}(\kappa)^{*}$, respectively. Then by applying $\tilde{L}_{\mathbf{c}(\kappa), b}^{(-1)}$ to both hand sides, we obtain the formula of the statement with $(j+1)$. 
By the continuity of the imbedding of $H_{0}^{1}(\Omega)$ into $H^{-1}(\Omega)$, and by Proposition 4 and by standard calculus in Banach space, we readily deduce the following.

Corollary 1 Let $\kappa \in \mathbb{C}$. Let $\Omega$ be a bounded open Lipschitz subset of $\mathbb{R}^{n}$. Let $\mathcal{W}$ be an open subset of $\mathbb{D}_{n}^{+}(\mathbb{R})$ such that condition $(19)$ holds true. Let $\left(b^{\sharp}, f^{\sharp}\right) \in \mathcal{W} \times H_{0}^{1}(\Omega)$. Then there exists $r \in] 0,+\infty\left[\right.$ such that $\operatorname{cl} \mathbb{B}_{\mathbb{D}_{n}(\mathbb{R})}\left(b^{\sharp}, r\right) \subseteq \mathcal{W}$ and such that

$$
\begin{aligned}
\Phi_{\mathbf{c}(\kappa), 1}[b, f]= & \Phi_{\mathbf{c}(\kappa), 1}\left[b^{\sharp}, f^{\sharp}\right]+\tilde{L}_{\mathbf{c}(\kappa), b^{\sharp}}^{(-1)}\left[f-f^{\sharp}\right] \\
& +\sum_{j=1}^{\infty}(-1)^{j}\left(\tilde{L}_{\mathbf{c}(\kappa), b^{\sharp}}^{(-1)} \circ \tilde{L}_{\mathbf{c}(\kappa)^{*}, b-b^{\sharp}}\right)^{j}\left[\phi_{\mathbf{c}(\kappa), f^{\sharp}}^{\times}\left(b^{\sharp}\right)\right],
\end{aligned}
$$

for all $(b, f) \in \mathbb{B}_{\mathbb{D}_{n}(\mathbb{R})}\left(b^{\sharp}, r\right) \times H_{0}^{1}(\Omega)$, where the series is normally convergent in $H_{0}^{1}(\Omega)$.

Now the right-hand side of (35) delivers a (nonlinear) analytic map from the set $\mathbb{B}_{\mathbb{D}_{n}(\mathbb{R})}\left(b^{\sharp}, r\right) \times H_{0}^{1}(\Omega)$ to $H_{0}^{1}(\Omega)$ and we know that $H_{0}^{1}(\Omega)$ is contained in both $\tilde{H}^{k-2}(\Omega)$ and $\tilde{H}^{k}(\Omega)$ when $k<1$ is an integer, and we ask whether there exists a nonlinear analytic map from $\mathbb{B}_{\mathbb{D}_{n}(\mathbb{R})}\left(b^{\sharp}, r\right) \times \tilde{H}^{k-2}(\Omega)$ to $\tilde{H}^{k}(\Omega)$ which extends the right-hand side of (35) perhaps for a smaller $r$ and when $k<1$ is an integer.

The idea is to replace the operator "factor" $\left(\tilde{L}_{\mathbf{c}(\kappa), b^{\sharp}}^{(-1)} \circ \tilde{L}_{\mathbf{c}(\kappa)^{*}, b-b^{\sharp}}\right)$ which appears in the right-hand side of (35) and which maps $H_{0}^{1}(\Omega)$ to itself by a linear and continuous map from $\tilde{H}^{k}(\Omega)$ to $\tilde{H}^{k}(\Omega)$ which extends the restriction of $\left(\tilde{L}_{\mathbf{c}(\kappa), b^{\sharp}}^{(-1)} \circ \tilde{L}_{\mathbf{c}(\kappa)^{*}, b-b^{\sharp}}\right)$ to $H_{0}^{1}(\Omega)$, and to show that the corresponding "replacement" series converges normally in $\tilde{H}^{k}(\Omega)$ for a perhaps smaller $r$. Since such a series is in the form of a power series in the variable $b$, its sum is real analytic. Then we observe that the sum of the first two terms in the right-hand side of (35) equals $\tilde{L}_{\mathbf{c}(\kappa), b^{\sharp}}^{(-1)}[f]$ and can be extended to a linear and continuous map from $\tilde{H}^{k-2}(\Omega)$ to $\tilde{H}^{k}(\Omega)$ of the variable $f$. Hence the sum of such extension and of the "replacement series" delivers an analytic function which equals the left-hand side of (35) if we choose the argument $(b, f)$ in $\mathbb{B}_{\mathbb{D}_{n}(\mathbb{R})}\left(b^{\sharp}, r\right) \times H_{0}^{1}(\Omega)$ (for the smaller $r$ ).

To do so, we need the following technical statement. For the convenience of the reader, we include a proof.

Theorem 9 Let $\mathbf{c} \in \mathcal{E}_{0}$. Let $\Omega$ be a bounded open Lipschitz subset of $\mathbb{R}^{n}, b \in \mathbb{D}_{n}^{+}(\mathbb{R})$. Let $\tilde{L}_{\mathbf{c}, b}$ be a linear homeomorphism from $H_{0}^{1}(\Omega)$ onto $H^{-1}(\Omega)$. Then the following statements hold.

(i) Let $k \in \mathbb{N} \backslash\{0\}$. If $\Omega$ is of class $C^{k-1,1}$, then $\tilde{L}_{\mathbf{c}, b}$ is a linear homeomorphism from $H^{k}(\Omega) \cap H_{0}^{1}(\Omega)$ onto $H^{k-2}(\Omega)$.

(ii) Let $k \in \mathbb{Z}, k \leq 0$. If $\Omega$ is of class $C^{1-k, 1}$, then there exists $\left.c_{k} \in\right] 0,+\infty[$ such that

$$
\left\|\tilde{L}_{\mathbf{c}, b}^{(-1)}[f]\right\|_{\tilde{H}^{k}(\Omega)} \leq c_{k}\|f\|_{\tilde{H}^{k-2}(\Omega)} \quad \forall f \in H_{0}^{1}(\Omega) .
$$

In particular there exists a unique linear and continuous operator $T_{\mathbf{c}, b, k}$ from $\tilde{H}^{k-2}(\Omega)$ to $\tilde{H}^{k}(\Omega)$ which extends the restriction to $H_{0}^{1}(\Omega)$ of $\tilde{L}_{\mathbf{c}, b}^{(-1)}$.

Proof Since $\tilde{L}_{\mathbf{c}, b}$ is a linear homeomorphism from $H_{0}^{1}(\Omega)$ onto $H^{-1}(\Omega)$, statement (i) follows by classical elliptic regularity theory (cf. e.g., Lions and Magenes [21, Thm. 5.4, p. 165]). We now turn to prove statement (ii) by transposition. Since $k \leq 0$, we have $2-k \geq 2$ 
and statement (i) implies that $\tilde{L}_{\mathbf{c}, b}$ is a linear homeomorphism from $H^{2-k}(\Omega) \cap H_{0}^{1}(\Omega)$ onto $H^{-k}(\Omega)$. Since $\Omega$ is a Lipschitz subset of $\mathbb{R}^{n}$, the space $\tilde{H}^{s}(\Omega)$ coincides with the subspace

$$
H_{\mathrm{cl} \Omega}^{s} \equiv\left\{u \in H^{s}\left(\mathbb{R}^{n}\right): \operatorname{supp} u \subseteq \operatorname{cl} \Omega\right\},
$$

of $H^{s}\left(\mathbb{R}^{n}\right)$, for each $s \in \mathbb{R}$, and the map from $H_{\mathrm{cl} \Omega}^{s}=\tilde{H}^{s}(\Omega)$ to the dual $\left(H^{-s}(\Omega)\right)^{\prime}$, which takes $v \in H_{\mathrm{cl} \Omega}^{s}$ to the element of $\left(H^{-s}(\Omega)\right)^{\prime}$ defined by

$$
<v, u>\equiv \int_{\mathbb{R}^{n}} \hat{v} \hat{U} \mathrm{~d} x \quad \forall u \in H^{-s}(\Omega),
$$

where $U$ is any element of $H^{-s}\left(\mathbb{R}^{n}\right)$ such that $U_{\mid \Omega}=u$ is a linear isometry for each $s \in \mathbb{R}$ (cf. e.g., McLean [23, Thm 3.14 (i), Thm. 3.29]). Thus $\tilde{H}^{k-2}(\Omega)$ is homeomorphic to the dual of $H^{2-k}(\Omega)$ and $\tilde{H}^{k}(\Omega)$ is homeomorphic to the dual of $H^{-k}(\Omega)$. Next we set

$$
\left(H^{2-k}(\Omega) \cap H_{0}^{1}(\Omega)\right)^{o} \equiv\left\{v \in\left(H^{2-k}(\Omega)\right)^{\prime}:<v, u>=0 \forall u \in H^{2-k}(\Omega) \cap H_{0}^{1}(\Omega)\right\},
$$

and we denote by $\pi$ the canonical projection from $\left(H^{2-k}(\Omega)\right)^{\prime}$ onto the quotient space $\left(H^{2-k}(\Omega)\right)^{\prime} /\left(H^{2-k}(\Omega) \cap H_{0}^{1}(\Omega)\right)^{o}$, and by $j$ the inclusion of $H^{2-k}(\Omega) \cap H_{0}^{1}(\Omega)$ into $H^{2-k}(\Omega)$. Then it is known that the transpose map $j^{t}$ is linear and continuous from $\left(H^{2-k}(\Omega)\right)^{\prime}$ to $\left(H^{2-k}(\Omega) \cap H_{0}^{1}(\Omega)\right)^{\prime}$, and that

$$
\operatorname{Ker} j^{t}=\left(H^{2-k}(\Omega) \cap H_{0}^{1}(\Omega)\right)^{o},
$$

and that the unique linear map $\left(j^{t}\right)^{\sim}$ such that

$$
j^{t}=\left(j^{t}\right)^{\sim} \circ \pi
$$

is a linear homeomorphism from $\left(H^{2-k}(\Omega)\right)^{\prime} /\left(H^{2-k}(\Omega) \cap H_{0}^{1}(\Omega)\right)^{o}$ onto the dual $\left(H^{2-k}(\Omega) \cap H_{0}^{1}(\Omega)\right)^{\prime}$. Now the transpose map $\left(\tilde{L}_{\mathbf{c}, b}^{(-1)}\right)^{t}$ is a linear homeomorphism from $\left(H^{2-k}(\Omega) \cap H_{0}^{1}(\Omega)\right)^{\prime}$ onto $\left(H^{-k}(\Omega)\right)^{\prime}$. Hence the composite map

$$
\left(\tilde{L}_{\mathbf{c}, b}^{(-1)}\right)^{t} \circ\left(j^{t}\right)^{\sim} \circ \pi=\left(\tilde{L}_{\mathbf{c}, b}^{(-1)}\right)^{t} \circ j^{t}
$$

is linear and continuous from $\left(H^{2-k}(\Omega)\right)^{\prime}$ to $\left(H^{-k}(\Omega)\right)^{\prime}$, which are canonically isomorphic to $\tilde{H}^{k-2}(\Omega)$ and to $\tilde{H}^{k}(\Omega)$, respectively. In particular, there exists $\left.c_{k} \in\right] 0,+\infty[$ such that

$$
\left\|\left(\tilde{L}_{\mathbf{c}, b}^{(-1)}\right)^{t} \circ j^{t}[f]\right\|_{\tilde{H}^{k}(\Omega)} \leq c_{k}\|f\|_{\tilde{H}^{k-2}(\Omega)} \quad \forall f \in H_{0}^{1}(\Omega),
$$

with the due canonical identifications. Thus statement (ii) follows by the equality

$$
\left(\tilde{L}_{\mathbf{c}, b}^{(-1)}\right)^{t} \circ j^{t}[f]=\tilde{L}_{\mathbf{c}, b}^{(-1)}[f] \quad \forall f \in H_{0}^{1}(\Omega),
$$

which we now turn to prove. Let $f \in H_{0}^{1}(\Omega)$. It suffices to show that if $u \in H^{-k}(\Omega)$, then

$$
<\left(\tilde{L}_{\mathbf{c}, b}^{(-1)}\right)^{t} \circ j^{t}[f], u>=<\tilde{L}_{\mathbf{c}, b}^{(-1)}[f], u>.
$$

Now we have

$$
<\left(\tilde{L}_{\mathbf{c}, b}^{(-1)}\right)^{t} \circ j^{t}[f], u>=<j^{t}[f], \tilde{L}_{\mathbf{c}, b}^{(-1)}[u]>=<f, j\left[\tilde{L}_{\mathbf{c}, b}^{(-1)}[u]\right]>=<f, \tilde{L}_{\mathbf{c}, b}^{(-1)}[u]>,
$$


by definition of transpose map. Indeed, $j$ is an inclusion map. By statement (i) with $k$ replaced by 2 and by $2-k$, and by the membership of $f$ in $H_{0}^{1}(\Omega) \subseteq L^{2}(\Omega)$ and of $u$ in $H^{-k}(\Omega)$, we know that

$$
\xi \equiv \tilde{L}_{\mathbf{c}, b}^{(-1)}[f] \in H^{2}(\Omega) \cap H_{0}^{1}(\Omega), \quad v \equiv \tilde{L}_{\mathbf{c}, b}^{(-1)}[u] \in H^{2-k}(\Omega) \cap H_{0}^{1}(\Omega) .
$$

Then we have

$$
<f, \tilde{L}_{\mathbf{c}, b}^{(-1)}[u]>=<\tilde{L}_{\mathbf{c}, b}[\xi], v>=\int_{\Omega} \tilde{L}_{\mathbf{c}, b}[\xi] v \mathrm{~d} x .
$$

Since both $\xi$ and $v$ belong to $H^{2}(\Omega)$ and have trace equal to 0 on $\partial \Omega$, the second Green identity implies that

$$
\int_{\Omega} \tilde{L}_{\mathbf{c}, b}[\xi] v \mathrm{~d} x=\int_{\Omega} \xi \tilde{L}_{\mathbf{c}, b}[v] \mathrm{d} x=<\xi, \tilde{L}_{\mathbf{c}, b}[v]>=<\tilde{L}_{\mathbf{c}, b}^{(-1)}[f], u>,
$$

(cf. e.g., McLean [23, Lem. 4.1, p. 114]). Hence, equality (36) holds true and the proof is complete.

We are now ready for the following.

Proof (of statement (iii) of Theorem 2) Clearly,

$$
\begin{aligned}
& \left\|\tilde{L}_{\mathbf{c}(\kappa)^{*}, v}[u]\right\|_{\tilde{H}^{k-2}(\Omega)}=\left\|\sum_{j=1}^{n} v_{j j} \frac{\partial^{2}}{\partial x_{j}^{2}} u\right\|_{\tilde{H}^{k-2}(\Omega)} \leq n|v|\|u\|_{\tilde{H}^{k}(\Omega)} \\
& \forall(v, u) \in \mathbb{D}_{n}(\mathbb{R}) \times \tilde{H}^{k}(\Omega),
\end{aligned}
$$

and accordingly,

$$
\left\|\tilde{L}_{\mathbf{c}(\kappa)^{*}, v}\right\|_{\mathcal{L}\left(\tilde{H}^{k}(\Omega), \tilde{H}^{k-2}(\Omega)\right)} \leq n r \quad \forall v \in \mathbb{B}_{\mathbb{D}_{n}(\mathbb{R})}(0, r),
$$

for all $r \in] 0,+\infty$ [. By Theorem 9 (ii), the operator $T_{\mathbf{c}(\kappa), b, k}$ is linear and continuous from $\tilde{H}^{k-2}(\Omega)$ to $\tilde{H}^{k}(\Omega)$ for all $b \in \mathcal{W}$. Thus if we choose $\left.r \in\right] 0,+\infty[$ as in Corollary 1 and such that $r<2^{-1} n^{-1}\left\|T_{\mathbf{c}(\kappa), b, k}\right\|_{\mathcal{L}\left(\tilde{H}^{k-2}(\Omega), \tilde{H}^{k}(\Omega)\right)}^{-1}$, then we have

$$
\begin{aligned}
& \left\|T_{\mathbf{c}(\kappa), b, k} \circ \tilde{L}_{\mathbf{c}(\kappa)^{*}, v}\right\|_{\mathcal{L}\left(\tilde{H}^{k}(\Omega), \tilde{H}^{k}(\Omega)\right)} \\
& \quad \leq\left\|T_{\mathbf{c}(\kappa), b, k}\right\|_{\mathcal{L}\left(\tilde{H}^{k-2}(\Omega), \tilde{H}^{k}(\Omega)\right)}\left\|\tilde{L}_{\mathbf{c}(\kappa)^{*}, v}\right\|_{\mathcal{L}\left(\tilde{H}^{k}(\Omega), \tilde{H}^{k-2}(\Omega)\right)} \\
& \quad \leq\left\|T_{\mathbf{c}(\kappa), b, k}\right\|_{\mathcal{L}\left(\tilde{H}^{k-2}(\Omega), \tilde{H}^{k}(\Omega)\right)} n r<2^{-1} \quad \forall v \in \mathbb{B}_{\mathbb{D}_{n}(\mathbb{R})}(0, r) .
\end{aligned}
$$

Hence, the membership of $\phi_{\mathbf{c}(\kappa), f^{\sharp}}^{\times}\left(b^{\sharp}\right)$ in $H_{0}^{1}(\Omega)=\tilde{H}^{1}(\Omega) \subseteq \tilde{H}^{k}(\Omega)$ implies that the power series in the right-hand side of (35) with $\left(\tilde{L}_{\mathbf{c}(\kappa), b^{\sharp}}^{(-1)} \circ \tilde{L}_{\mathbf{c}(\kappa)^{*}, b-b^{\sharp}}\right)$ replaced by $\left(T_{\mathbf{c}(\kappa), b^{\sharp}, k} \circ \tilde{L}_{\mathbf{c}(\kappa)^{*}, b-b^{\sharp}}\right)$ is normally convergent in $\tilde{H}^{k}(\Omega)$ for all $b \in \mathbb{B}_{\mathbb{D}_{n}(\mathbb{R})}\left(b^{\sharp}, r\right)$. Accordingly, the map $\tilde{\Phi}_{\mathbf{c}(\kappa), k, b^{\sharp}, f^{\sharp}}$ from $\mathbb{B}_{\mathbb{D}_{n}(\mathbb{R})}\left(b^{\sharp}, r\right) \times \tilde{H}^{k-2}(\Omega)$ to $\tilde{H}^{k}(\Omega)$ defined by

$$
\begin{aligned}
\tilde{\Phi}_{\mathbf{c}(\kappa), k, b^{\sharp}, f^{\sharp}}[b, f] \equiv & T_{\mathbf{c}(\kappa), b^{\sharp}, k}\left[f^{\sharp}\right]+T_{\mathbf{c}(\kappa), b^{\sharp}, k}\left[f-f^{\sharp}\right] \\
+ & \sum_{j=1}^{\infty}(-1)^{j}\left(T_{\mathbf{c}(\kappa), b^{\sharp}, k} \circ \tilde{L}_{\mathbf{c}(\kappa)^{*}, b-b^{\sharp}}\right)^{j}\left[\phi_{\mathbf{c}(\kappa), f^{\sharp}}^{\times}\left(b^{\sharp}\right)\right] \\
& \forall(b, f) \in \mathbb{B}_{\mathbb{D}_{n}(\mathbb{R})}\left(b^{\sharp}, r\right) \times \tilde{H}^{k-2}(\Omega),
\end{aligned}
$$


is real analytic. Since $\Phi_{\mathbf{c}(\kappa), 1}\left[b^{\sharp}, f\right]=T_{\mathbf{c}(\kappa), b^{\sharp}, k}[f]$ for all $f \in H_{0}^{1}(\Omega)$, then Corollary 1 ensures that equality (20) holds true.

\section{References}

1. Ammari, H., Kang, H.: Polarization and Moment Tensors with Applications to Inverse Problems and Effective Medium Theory. Springer, New York (2007)

2. Ammari, H., Kang, H., Lim, M.: Effective parameters of elastic composites. Indiana Univ. Math. J. 55, 903-922 (2006)

3. Ammari, H., Kang, H., Touibi, K.: Boundary layer techniques for deriving the effective properties of composite materials. Asymptot. Anal. 41, 119-140 (2005)

4. Arens, T., Sandfort, K., Schmitt, S., Lechleiter, A.: Analysing Ewald's method for the evaluation of Green's functions for periodic media. IMA J. Appl. Math. 78, 405-431 (2013)

5. Berman, C.L., Greengard, L.: A renormalization method for the evaluation of lattice sums. J. Math. Phys. 35(2), 6036-6048 (1994)

6. Böhme, R., Tomi, L.: Zur Struktur der Lösungsmenge des Plateauproblems. Math. Z. 133, 1-29 (1973)

7. Dalla Riva, M.: A family of fundamental solutions of elliptic partial differential operators with real constant coefficients. Integral Equ. Oper. Theory 76, 1-23 (2013)

8. Dalla Riva, M., Lanza de Cristoforis, M., Musolino, P.: Analytic dependence of volume potentials corresponding to parametric families of fundamental solutions. Integral Equ. Oper. Theory 82, 371-393 (2015)

9. Dalla Riva, M., Morais, J., Musolino, P.: A family of fundamental solutions of elliptic partial differential operators with quaternion constant coefficients. Math. Methods Appl. Sci. 36, 1569-1582 (2013)

10. Dalla Riva, M., Musolino, P.: A singularly perturbed nonlinear traction problem in a periodically perforated domain: a functional analytic approach. Math. Methods Appl. Sci. 37, 106-122 (2014)

11. Deimling, K.: Nonlinear Functional Analysis. Springer, Berlin (1985)

12. Folland, G.B.: Introduction to Partial Differential Equations. Princeton University Press, Princeton (1995)

13. Gilbarg, D., Trudinger, N.S.: Elliptic Partial Differential Equations of Second Order. Springer, Berlin (1983)

14. Henry, D.: Topics in Nonlinear Analysis. Trabalho de Matemática, vol. 192, Brasilia (1982)

15. Lanza de Cristoforis, M.: Properties and pathologies of the composition and inversion operators in Schauder spaces. Acc. Naz. delle Sci. detta dei XL 15, 93-109 (1991)

16. Lanza de Cristoforis, M., Musolino, P.: A perturbation result for periodic layer potentials of general second order differential operators with constant coefficients. Far East J. Math. Sci. 52, 75-120 (2011)

17. Lanza de Cristoforis, M., Musolino, P.: A real analyticity result for a nonlinear integral operator. J. Integral Equ. Appl. 25, 21-46 (2013)

18. Lanza de Cristoforis, M., Musolino, P.: A quasi-linear heat transmission problem in a periodic two-phase dilute composite. A functional analytic approach. Comm. Pure Appl. Anal. 13, 2509-2542 (2014)

19. Lanza de Cristoforis, M., Rossi, L.: Real analytic dependence of simple and double layer potentials upon perturbation of the support and of the density. J. Integral Equ. Appl. 16, 137-174 (2004)

20. Lin, C.S., Wang, C.L.: Elliptic functions, Green functions and the mean field equations on tori. Ann. Math. 172, 911-954 (2010)

21. Lions, J.L., Magenes, E.: Non-homogeneous Boundary Value Problems and Applications, vol. I. Springer, New York (1972)

22. Mamode, M.: Fundamental solution of the Laplacian on flat tori and boundary value problems for the planar Poisson equation in rectangles. Bound. Value Probl. 2014, 221 (2014)

23. McLean, W.: Strongly Elliptic Systems and Boundary Integral Equations. Cambridge University Press, Cambridge (2000)

24. Mityushev, V., Adler, P.M.: Longitudinal permeability of spatially periodic rectangular arrays of circular cylinders. I. A single cylinder in the unit cell. ZAMM Z. Angew. Math. Mech. 82, 335-345 (2002)

25. Schmeisser, H.J., Triebel, H.: Topics in Fourier Analysis and Function Spaces. Akademische Verlagsgesellschaft Geest \& Portig K.-G, Leipzig (1987)

26. Tornberg, A.K., Greengard, L.: A fast multipole method for the three-dimensional Stokes equations. J. Comput. Phys. 227, 1613-1619 (2008)

27. Triebel, H.: Theory of Function Spaces. Birkhäuser Verlag, Basel (1983)

28. Valent, T.: Boundary Value Problems of Finite Elasticity. Local Theorems on Existence, Uniqueness and Analytic Dependence on Data. Springer, New York (1988) 